\title{
Bitumen-Bearing Deposits of the United States uwco Libray
} DEC 281988 Depository Shipment

\section{4}

\section{U.S. GEOLOGICAL SURVEY BULLETIN 1784}





\section{Bitumen-Bearing Deposits of the United States}

By Bonnie L. Crysdale and Christopher J. Schenk

A summary of the locations, resources,

and petrophysical properties of

bitumen-bearing rocks in the United States 


\section{DEPARTMENT OF THE INTERIOR \\ DONALD PAUL HODEL, Secretary}

\section{U.S. GEOLOGICAL SURVEY}

Dallas L. Peck, Director

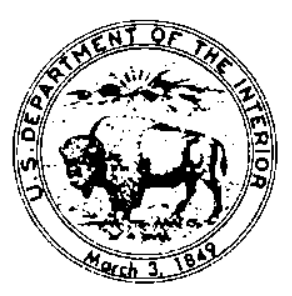

For sale by the Books and Open-File Reports Section, U.S. Geological Survey, Federal Center,

Box 25425, Denver, CO 80225

Library of Congress Cataloging in Publication Data

Crysdale, Bonnie L.

Bitumen-bearing deposits of the United States.

(U.S. Geological Survey bulletin ; 1784)

Bibliography: $p$.

1. Bitumen-United States. 2. Bitumen-Testing. I. Schenk, Christopher

J. II. Title. III. Series.

QE75.B9 no. $1784 \quad 557.3$ s $\quad 87-600085$

[TN850] [553.2'7] 


\title{
CONTENTS
}

\author{
Abstract 1 \\ Introduction 1 \\ References 2
}

\section{PLATES}

[Plates in pocket]

1. Map showing locations of bitumen-bearing deposits in the conterminous United States

2. Map showing locations of bitumen-bearing deposits in Alaska

\section{TABLES}

1-17. Summary of bitumen deposits by State:
1. Alabama
2. Alaska 8
3. Arizona 10
4. Arkansas 12
5. California 14
6. Colorado 24
7. Kentucky $\mathbf{2 4}$
8. Michigan 26
9. Mississippi 26
10. Montana 26
11. New Mexico 28
12. New York 28
13. Ohio 28
14. Oklahoma $\mathbf{3 0}$
15. Texas 32
16. Utah $\mathbf{3 4}$
17. Wyoming 



\title{
Bitumen-Bearing Deposits of the United States
}

\author{
By Bonnie L. Crysdale and Christopher J. Schenk
}

\begin{abstract}
Tar sands, or bitumen-bearing rocks, represent a significant source of hydrocarbons in the United States. This report is a summary, by State, of the locations, resources, and petrophysical data of known bitumen-bearing deposits. Bitumen accumulations are located primarily in Alabama, California, Kentucky, Texas, and Utah. Utah contains the largest resource, with an estimated 28 billion barrels of bitumen, followed by California with 9 billion barreis, and Alabama, with approximately 6 billion barrels. The locations of most bitumen accumulations are well known, although the resources of only the larger deposits have been estimated. Much of the petrophysical, chemical, and mineralogical data are unknown, even for the larger deposits.
\end{abstract}

\section{INTRODUCTION}

The purpose of this report is to summarize the locations and resources of known tar sand deposits in the United States (pls. 1,2). Tar sand is a generic term for a sedimentary rock or sand containing a heavy asphaltic substance called bitumen (Schramm, 1979), which in its natural state is not mobile at reservoir conditions and cannot be extracted by conventional petroleum recovery methods. Tar, or bitumen, is defined as any hydrocarbon deposit with a gas-free viscosity greater than 10,000 centipoises (cp) measured at original reservoir conditions (Danyluk and others, 1984), and density greater than $1,000 \mathrm{~kg} / \mathrm{m} 3\left(\left(10^{\circ}\right.\right.$ API gravity) at $15.6^{\circ} \mathrm{C}$ $\left(60^{\circ} \mathrm{F}\right)$ at atmospheric pressure. Viscosity data for many of the deposits generally are not available, so bitumen as used in this report is defined by the API gravity cutoff of less than $10^{\circ}$. Bitumen-bearing rocks are known by scveral other names, such as natural asphalt, tarry oil, bituminous rock, tar sands, oil sands, and rock asphalt. All appear to be used interchangeably. We will use the term bitumen-bearing to describe these accumulations. Host lithologies of the various bitumen-bcaring rocks include sandstone, limestone, dolomite, conglomerate, siltstone, and unconsolidated sands. Most of the bitumen-bearing rocks are located on the margins of sedimentary basins, and have outcrop or surface exposures, although they do extend into the subsurface.
Bitumen-bearing rocks have been recognized in the United States since the late 1800's; Ball Associates (1965) listed over 500 occurrences, most of them small, isolated outcrops. Lewin and Associates (1984) reassessed the bitumen resource, estimating the total at 54 billion barrels, with 25 major (greater than 100 million barrels) and 19 minor (10 to 100 million barrels) occurrences. Lewin and Associates (1984) categorized slightly less than half of this amount ( 22 billion barrels) as reserves and considered the remainder to be speculative resource. Deposits occur primarily in Alabama, California, Kentucky, Texas, and Utah. Utah contains the largest deposits in terms of resource, size, and number, with an estimated 28 billion barrels of bitumen on more than 700,000 acres of land. Accumulations in the tristate area of Missouri, Kansas, and Oklahoma (shown on pl. 1, but not numbered) are estimated to contain approximately 3 billion barrels of petroleum (Lewin and Associates, 1984); however, they are not listed in this report as most of these accumulations appear to consist of heavy oil (Ebanks and Weber, 1984). Crude oils with densities from 934 to $1,000 \mathrm{~kg} / \mathrm{m}^{3}$ (API gravities of $20^{\circ}$ to $10^{\circ}$ ) inclusive are classified as heavy oils (Martinez, 1984). Similarly, the giant Kuparuk deposit on the Alaska North Slope is now known to consist mainly of heavy oil, not bitumen (Werner, 1984), with a resource estimated as high as 40 billion barrels. Lewin and Associates considered 10 billion barrels of the Kuparuk deposit to be bitumen; consequently, their total bitumen resource estimate of 54 billion barrels should be adjusted downward to 44 billion barrels.

Bitumen-bearing rocks were quarried extensively for road-paving material in the United States from the late 1800 's through the 1940 's, until it became uneconomic to continue mining operations. Bitumenbearing rocks have been recognized for many years as potential energy sources (Schramm, 1979); however, interest has remained marginal due to (1) unfavorable economic conditions for extracting the bitumen from the rock, (2) limited knowledge of the character of bitumenbearing deposits, and (3) large, readily available reserves 
of conventional petrolcum. Recent interest has centered on developing methods of in-situ recovery of the bitumen.

The bitumen deposits summarized in tables 1 through 17 are listed by State and generally coincide with field outlines of major deposits described by Lewin and Associates (1984) and minor deposits characterized by Ball Associates (1965). All map location numbers in the tables refer to plate 1, except the table for Alaska, which refers to plate 2. Descriptions in the tables include the following information: (1) map number located on the plates by State (where numbered $1 \mathrm{~A}, 1 \mathrm{~B}$, and so on, the numbers indicate separate occurrences within onc outlined area); (2) name of the deposit and its location; (3) geologic formation containing the bitumen, age of the formation, and lithology of the host rock; (4) depth to pay, and pay thickness; (5) number of acres the deposit covers, cither measured (by well control and core analysis) or speculative (by tar shows and geologic interpretations); (6) API gravity and viscosity of the bitumen; (7) porosity and permeability of the host rock; (8) water saturation (in percent) and bitumen saturation (cither percent or weight percent) of the host rock; (9) sulfur content of the bitumen (bitumens generally contain 3 weight percent or more of sulfur (Danyluk and others, 1984)); and (10) amount of bitumen resource in place, both measurcd and speculative. Plates 1 and 2 also illustrate the oullines of sedimentary basins in the conterminous United States and Alaska. Basin outlines for the conterminous United States are from Terra Graphics (1977), and basin outlines for Alaska are from Ehm (1983). Data presented here are based on the most reliable published and unpublished information available from various Fedcral, State and local agencies, private sources, and original field investigations.

\section{REFERENCES}

Adams, E. W., and Beatty, W. B., 1962, Bituminous rocks in California: Division of Mines and Geology, Mineral Information Service, v. 15, no. 4, p. 1-11.

Ball Associates, Ltd., 1965, Surface and shallow oil impregnated rocks and shallow oil fields in the United States: U.S. Bureau of Mines, Monograph 12, $375 \mathrm{p}$

Blasko, P. P., 1976a, Oil and gas seeps in Alaska; north-central Gulf of Alaska: Bureau of Mines Report of Investigations RI-8136, p. $102-105$.

1976b, Oil and gas seeps in Alaska, Alaska Peninsula; western Gulf of Alaska: Bureau of Mines Report of Investigations RI-8122, $78 \mathrm{p}$.

Britton, M. W., 1984, Problems frequently encountered in cvaluating tar sand resources-example: the south Texas San Miguel deposit: American Association of Petroleum Geologists Research Conference, Exploration for Heavy Crude Oil and Bitumen, Santa Maria, Calif,, Preprint, 24 p.
California Division of Oil and Gas, 1974, California oil and gas fields, volume 2, South, central coastal, and offshore California: Report no. TR12. [ unnumbered pages].

Campbell, J. A., and Ritzma, H. R., 1979, Gcology and petroleum resources of the major oil-impregnated sandstone deposits of Utah: Utah Geological and Mineral Survey Special Studies 50, 24 p.

Clardy, B. F., 1983, unpublished memorandum to N. F. Williams, Arkansas Gcological Commission, Little Rock, Arkansas.

Danyluk, M. D., Galbraith, B. E., and Omana, R. A., 1984, Toward definitions for heavy crude oils and tar sands, in Meyer, R. F., Wynn, J. C., and Olson, J. C., eds., The future of heavy crude and tar sands: UNITAR Second International Conference, p. 1xvii-1xviii.

deChadenedes, Francois, 1984, Surface and tar sand deposits in California: American Association of Petroleum Geologists Research Conference, Exploration for Heavy Crude Oil and Bitumen, Santa Maria, Calif., v. 1, 14 p.

Ebanks, W. J., and Weber, J. F., 1984, Shallow heavy-oil deposit in a Pennsylvanian fluvial sandstone reservoir, Eastburn Field, Missouri, U.S.A.: American Association of Petroleum Geologists Research Conference, Exploration for Heavy Crude Oil and Bitumen, Santa Maria, Calif., v. $1,35 \mathrm{p}$

Elım, Arlen, 1983, Oil and gas basins map of Alaska: Alaska Department of Natural Resources, 1:2,500,000, 1 sheet.

Evans, T. J., 1975, Native bituminous materials in Texas: Texas Bureau of Economic Geology, Mineral Resources Circular no. 57, p. 1-11.

Harrison, W. E., and Burchfield, M. R., 1984, Resource evaluation of selected tar-sand deposits in southern Oklahoma: American Association of Petroleum Geologists Rescarch Conference, Exploration for Heavy Crude Oil and Bitumen, Santa Maria, Calif,, v. 1, 41 p.

Harrison, W. E., Mankin, C. J., Weber, S. J., and Curiale, J. A., 1981, Oil-sand and heavey-oil potential of Oklahoma, in Meyer, R. F., and Steele, C. T., eds., The future of heavy crude oils and tar sands: UNITAR First International Conference, p. 83-89.

Jennings, C. W., 1957, Asphalt and bitutminous rock: Mineral Commodities of California, California Division of Mines Bullatin 176.

Lewin and Associates, Inc., 1984, Major tar sand and heavy oil deposits of the United States: Interstate Oil Compact Commission, 272 p.

Martinez, A. R., 1984, Report of working group on definitions, in Meyer, R. F., Wynn, J. C., and Olson, J. C., eds., The future of heavy crude and tar sands: UNITAR Second International Conference, p. kxvii-lxviii.

McGrain, Preston, 1976, Tar sands (rock asphalt) of Kentucky-a review: Kentucky Geological Survey, ser. 10, Report of Investigations 19, $16 \mathrm{p}$.

Molenaar, C. M., 1977, The Pinedale oil seep-an exluumed stratigraphic trap in the southwestern San Juan basin: New Mexico Geological Socicty Guidebook, 28th Field Conference, San Juan Basin III, p. 243-246.

Nager, M. C., 1984, Tar-sand exploration in Kentucky: Kentucky Geological Survey, Lexington, 51 p. 
Peterson, P. R., and Ritzma, H. R., 1974, Informational core drilling in Utah's oil-impregnated sandstone deposits, southeastern Uinta Basin, Uintah County, Utah: Utah Geological And Mineralogical Survey, Report of Investigation no. 88, p. 9-10.

Ritzma, H. R., 1968, Preliminary location map of oilimpregnated rock deposits of Utah: Urah Geological and Mineralogical Survey, Map no. 25.

1979, Oil-impregnated rock deposits of Utah: Utah Geological and Mineralogical Survey, Map 47, 2 sheets, 1:1,000,000.

Schramm, L. W., 1979, U.S. tar sand oil forecasts (1985-1995): U.S. Department of Energy Technical Report, 44 p.
Terra Graphics, 1977, Oil and gas production map of the United States. 1:3,168,000.

Werner, M. R., 1984, Shallow heavy oil deposits of the Kuparuk River unit: American Association of Petroleum Geologists Research Conference, Exploration for Heavy Crude Oil and Bitumen, Santa Maria, Calif., v. 2, 31 p.

Williams, D. A., 1982, Structural and geochemical study of the South Sulphur asphalt deposits, Murray County, Oklahoma: University of Oklahoma, M.S. thesis, $123 \mathrm{p}$.

Wilson, Gary V., 1984, Bitumen deposits of northwest Alabama: American Association of Petroleum Geologists Research Conference, Exploration for Heavy Crude Oil and Bitumen, Santa Maria, Calif., v. 2, 10 p. 



\section{TABLES 1-17}

\section{Abbreviations and symbols used in tables}

[Leaders (--) indicate no data available; do, ditto; M, measured; S, speculative; T, total; cp centipoise; md, millidarcy; m.b., thousand barrels; mi, mile; wt, weight; res. temp, reservoir temperature; @, at; *, average; ls, limestone; ss, sandstone; sh, shale; sts, siltstone; diat, diatomite; cgl, conglomerate; mds, mudstone; dol, dolomite; cls, claystone; qzt, quartzite; ark., arkose] 
Table 1. Summary of bitumen deposits in Alabama

\begin{tabular}{|c|c|c|c|c|c|c|c|c|}
\hline $\begin{array}{l}\text { Map } \\
\text { number }\end{array}$ & Deposit name & County & $\begin{array}{l}\text { Townships } \\
\text { and Ranges }\end{array}$ & Formation & Age & $\begin{array}{l}\text { Lith- } \\
\text { ology }\end{array}$ & $\begin{array}{l}\text { Depth } \\
(\mathrm{ft})\end{array}$ & $\begin{array}{l}\text { Pay } \\
\text { thickness } \\
\quad(\mathrm{ft})\end{array}$ \\
\hline $1 \mathrm{~A}$ & 2 Bangor & $\begin{array}{l}\text { Franklin, } \\
\text { Lawrence, } \\
\text { Morgan }\end{array}$ & $\begin{array}{l}\mathrm{l}_{\mathrm{T}} .7,8 \mathrm{~s}, \\
\mathrm{R} .3,5,9 \mathrm{~W} .\end{array}$ & $\begin{array}{l}\text { Bangor } \\
\text { Limestone }\end{array}$ & Mississippian & Is & outcrop & --- \\
\hline $1 \mathrm{~B}$ & ${ }^{2}$ Hartselle & $\begin{array}{l}\text { Colbert, } \\
\text { Franklin, } \\
\text { Lawrence, } \\
\text { Morgan, } \\
\text { Mar1on, } \\
\text { Winston, } \\
\text { Walker, } \\
\text { Cullman }\end{array}$ & $\begin{array}{l}\text { T. } 5-13 \mathrm{~S} ., \\
\mathrm{R} .4-15 \mathrm{~W} .\end{array}$ & $\begin{array}{l}\text { Hartselle } \\
\text { Sandstone }\end{array}$ & $---d o^{---}$ & ss & $0-1,000$ & $5-54$ \\
\hline $2 \mathrm{~A}$ & $\begin{array}{l}{ }^{1} \text { Colbert } \\
\text { Creek }\end{array}$ & Colbert & $\begin{array}{l}\text { T. } 3 \mathrm{~S} ., \\
\mathrm{R} .14 \mathrm{~W} .\end{array}$ & Girkin & $---\mathrm{do}---$ & $1 s$ & outcrop & --- \\
\hline $2 \mathrm{~B}$ & $1_{\text {Cherokee }}$ & $-\cdots$ do--- & $\begin{array}{l}\text { T. } 4 \mathrm{~S} ., \\
\text { R. } 13-14 \mathrm{~W} .\end{array}$ & $\begin{array}{l}--- \text { do--- } \\
\text { Bethe1 } \\
\text { Sandstone }\end{array}$ & $\rightarrow--\mathrm{do}-\infty$ & --- & --- & $3-12$ \\
\hline $2 \mathrm{C}$ & $1_{\text {Margerum }}$ & $-\cdots$ do $-\longrightarrow$ & $\begin{array}{l}\text { T. 3-5 S., } \\
\text { R. } 14-15 \text { W. }\end{array}$ & Girkin & --- do--- & is & --- & $<1-25$ \\
\hline $2 \mathrm{D}$ & $\begin{array}{l}{ }^{2} \text { Pride } \\
\text { Mountain }\end{array}$ & $---\mathrm{do-}--$ & --- & $\begin{array}{l}\text { Pride } \\
\text { Mountain }\end{array}$ & --- do-- & $\begin{array}{l}3 \text { sh, } \\
1 s, \\
\text { ss, } \\
\text { sts }\end{array}$ & $\begin{array}{r}20-30 \\
\text { mi } \\
\text { outcrop }\end{array}$ & I $2^{\star}$ \\
\hline
\end{tabular}

\footnotetext{
$1_{\text {Ball Associates, } 1965 .}$

${ }^{2}$ Lewin and Associates, 1984.

3 Wilson, 1984.
} 


\begin{tabular}{|c|c|c|c|c|c|c|c|c|}
\hline Acreage & $\begin{array}{c}\text { API } \\
\text { gravity } \\
\text { (degrees) }\end{array}$ & $\begin{array}{l}\text { Viscosity } \\
(c p)\end{array}$ & $\begin{array}{l}\text { Porosity } \\
(\%)\end{array}$ & $\begin{array}{l}\text { Permeability } \\
(\mathrm{md})\end{array}$ & $\begin{array}{c}\text { Water } \\
\text { sat. } \\
(\%)\end{array}$ & $\begin{array}{c}\text { Bitumen } \\
\text { sat. } \\
(\%)\end{array}$ & $\begin{array}{l}\text { Sulfur } \\
\text { (wt \%) }\end{array}$ & $\begin{array}{c}\text { Resource in } \\
\text { place } \\
\text { (m.b.) }\end{array}$ \\
\hline$-\cdots$ & --- & --- & --- & --- & --- & --- & -- & --- \\
\hline $\begin{array}{lr}M= & 534,000 \\
S= & 1,500,000 \\
T= & 2,034,000\end{array}$ & --- & --- & $6-24$ & $1-700$ & --- & 35 & $1.1-2.6$ & $\begin{array}{l}M=1,760,000 \\
S=4,500,000 \\
T=6,260,000\end{array}$ \\
\hline - & -- & --- & -- & - & --- & --- & -- & --- \\
\hline-- & --- & -- & -- & -- & -- & $3-14$ wt & --- & -- \\
\hline-- & -- & --- & - & --- & --- & $5-7$ wt & --- & --- \\
\hline $\mathrm{T}=25 \mathrm{mi}^{2}$ & $\longrightarrow$ & -- & --- & --- & --- & 6 & -- & $\begin{array}{l}\mathrm{S}=100,000 \\
\mathrm{~T}=100,000\end{array}$ \\
\hline
\end{tabular}


Table 2. Summary of bitumen deposits in Alaska

\begin{tabular}{|c|c|c|c|c|c|c|c|c|c|}
\hline $\begin{array}{l}\text { Map } \\
\text { number }\end{array}$ & Deposit name & Location & $\begin{array}{l}\text { ownships } \\
\text { Ind Ranges }\end{array}$ & Formation & Age & $\begin{array}{l}\text { Lith- } \\
\text { ology }\end{array}$ & $\begin{array}{l}\text { Depth } \\
\text { (ft) }\end{array}$ & $\begin{array}{r}P g \\
\text { thick } \\
\text { (f }\end{array}$ & $\begin{array}{l}\text { Pay } \\
\text { ckness } \\
\text { (ft) }\end{array}$ \\
\hline 1 & ${ }^{1}$ Li tuya Bay & $\begin{array}{l}110 \mathrm{~m} 1 \text { west } \\
\text { of Juneau }\end{array}$ & -- & -- & Tertfary & ss & --- & & -- \\
\hline 2 & $\begin{array}{l}{ }^{1} \text { Cape Kekur- } \\
\text { no1 (Cold } \\
\text { Bay) }\end{array}$ & $\begin{array}{l}\text { West shore of } \\
\text { Shelikof Strait }\end{array}$ & -- & --- & $\begin{array}{l}\text { Late } \\
\text { Triassic }\end{array}$ & $1 \mathrm{~s}$ & outcrop & & --- \\
\hline 3 & $\begin{array}{c}{ }^{1} \text { Chignik } \\
\text { Lagoon }\end{array}$ & $\begin{array}{l}\text { On east side of } \\
\text { Alaskan Penin- } \\
\text { sula }\end{array}$ & --- & Ch1gnik & Cretaceous & $\begin{array}{l}\text { ss, } \\
\text { cgi }\end{array}$ & --- & $\begin{array}{l}120 \mathrm{~s} \\
200 \mathrm{II} \\
\mathrm{ss} \mathrm{a}\end{array}$ & $\begin{array}{l}\text { ss, cgl } \\
\text { marine } \\
\text { and cgl }\end{array}$ \\
\hline 4 & ${ }^{1}$ Nation River & $\begin{array}{l}\text { On Yukon River, } \\
20 \mathrm{mi} \text {, west of } \\
\text { Canadian border }\end{array}$ & -- & - & $\begin{array}{l}\text { Mississ- } \\
\text { ippian }\end{array}$ & $1 \mathrm{~s}$ & outcrop & & $-\cdots$ \\
\hline 5 & $\begin{array}{l}\mathbf{l}_{\text {Tiglukpuk }} \\
\text { Creek }\end{array}$ & $\begin{array}{l}\text { On east side } \\
\text { of T1glukpuk } \\
\text { Creek, } 50 \mathrm{mi} \text {. } \\
\text { south of Umiat } \\
\text { ollfield }\end{array}$ & --- & $\begin{array}{l}\text { Okp1kruak, } \\
\text { Toruk }\end{array}$ & Cretaceous & $\begin{array}{l}\text { asphalt } \\
\text { in (?) }\end{array}$ & -ndom- & & -- \\
\hline 6 & $\begin{array}{l}{ }^{1} \text { Fortress } \\
\text { Mountain }\end{array}$ & $\begin{array}{l}\text { Between Aylyak } \\
\text { River and Okak } \\
\text { Creek, } 65 \text { m1. } \\
\text { south of Umiat } \\
\text { ollfield }\end{array}$ & -- & Toruk & $\begin{array}{l}\text { Early } \\
\text { Cretaceous }\end{array}$ & $\begin{array}{l}\mathrm{cgl} \\
\mathrm{ss} ?\end{array}$ & - & & -- \\
\hline 7 & $\begin{array}{l}{ }_{\text {L1 sburne }} \\
\text { Ridge }\end{array}$ & $\begin{array}{l}\text { Between Etivluk } \\
\text { and Kilfgwa River } \\
\text { 1n west Arctic } \\
\text { foothills }\end{array}$ & er & $\begin{array}{l}\text { Okpikruak, } \\
\text { Shublik, } \\
\text { Kiruktagial }\end{array}$ & $\begin{array}{l}\text { Early Cretaceous } \\
\text { Trlassic } \\
\text { Mississ- } \\
\text { Ipptan }\end{array}$ & $\begin{array}{l}\text { ss, } \\
\text { ls, } \\
\text { cherty } \\
\text { asphalt }\end{array}$ & --- & & --- \\
\hline 8 & $\begin{array}{l}{ }^{1} \text { Utukok } \\
\text { RI ver }\end{array}$ & $\begin{array}{l}\text { Near Utukok } \\
\text { River on } \\
\text { Carbon Cr. } \\
\text { anticline }\end{array}$ & $\begin{array}{l}\text { lat } 68^{\circ} \\
21^{-} 24^{\prime \prime} \mathrm{N} \\
\text { long } 160^{\circ} \\
04^{-}-30^{\prime \prime} \mathrm{W}\end{array}$ & Kukpowruk & $\begin{array}{l}\text { Early } \\
\text { Cretaceous }\end{array}$ & ss & outcrop & & -- \\
\hline 9 & ${ }^{1}$ Carbon $\mathrm{Cr}$. & $\begin{array}{l}6 \mathrm{mi} \text {. west } \\
\text { of Carbon } \mathrm{Cr} \text {. }\end{array}$ & $\begin{array}{l}\text { lat } 68^{\circ} \\
22^{-} 54^{\prime \prime} \mathrm{N} \\
\text { long } 159^{\circ} \\
59^{-3} 30^{\prime \prime W}\end{array}$ & Kukpowruk & $\begin{array}{l}\text { Early } \\
\text { Cretaceous }\end{array}$ & ss & outcrop & & -- \\
\hline 10 & $l_{\text {Omicron Hill }}$ & - & $\begin{array}{l}\text { lat } 69^{\circ} \\
29^{-3} 30^{\prime \prime} \mathrm{N} \\
\text { 1ong } 160^{\circ} \\
30^{-} 30^{\prime \prime} \mathrm{W}\end{array}$ & ---do--- & $\begin{array}{l}\text { Early } \\
\text { Cretaceous }\end{array}$ & ss & outcrop & & --- \\
\hline 11 & $\begin{array}{l}{ }^{1} \text { Kokolik } \\
\text { River }\end{array}$ & $\begin{array}{l}42 \mathrm{mi} \text {. from } \\
\text { river mouth }\end{array}$ & -- & Corwin & --- do--- & ss & -- & & 7 \\
\hline 12 & ${ }^{2}$ o1ly Lake & $\begin{array}{l}\text { Mt. St. Elias } \\
\text { quad. }\end{array}$ & $\begin{array}{l}\text { T. } 21 \text { S., } \\
\text { R. } 28 \mathrm{E} ., \\
\mathrm{N} 1 / 2 \\
\text { sec. } 6\end{array}$ & $\begin{array}{l}\text { impreg. } \\
\text { surface } \\
\text { deposits }\end{array}$ & --- & - & --- & & --- \\
\hline 13 & ${ }^{3}$ Oil Creek & Karluk quad. & $\begin{array}{l}\text { T. } 29 \mathrm{~S} ., \\
\text { R. } 40 \mathrm{~W} ., \\
\text { SE } 1 / 4 \\
\text { sec. } 10\end{array}$ & $---d o---$ & --- & --- & --- & & -- \\
\hline 14 & ${ }^{1}$ Humphrey & $\begin{array}{l}\text { Un-goon Pt. } \\
\text { on Arctic } \\
\text { coast }\end{array}$ & --- & 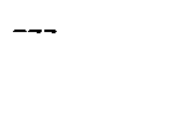 & -- & ss & --- & & 4 \\
\hline
\end{tabular}

l Ba11 Associates, 1965.

2 Bl asko, $1976 \mathrm{a}$.

3 Blasko, $1976 \mathrm{~b}$. 


\begin{tabular}{|c|c|c|c|c|c|c|c|c|}
\hline Acreage & $\begin{array}{c}\text { API } \\
\text { gravity } \\
\text { (degrees) }\end{array}$ & $\begin{array}{l}\text { Viscosity } \\
\text { (cp) }\end{array}$ & $\begin{array}{l}\text { Porosity } \\
(\%)\end{array}$ & $\begin{array}{l}\text { Permeability } \\
\text { (md) }\end{array}$ & $\begin{array}{l}\text { Water } \\
\text { sat. } \\
(\%)\end{array}$ & $\begin{array}{c}\text { Bitumen } \\
\text { sat. } \\
(\%)\end{array}$ & $\begin{array}{l}\text { Sulfur } \\
\text { (wt \%) }\end{array}$ & $\begin{array}{l}\text { Resource in } \\
\text { place } \\
\text { (m.b.) }\end{array}$ \\
\hline--- & --- & --- & -- & -- & -- & --- & -- & -- \\
\hline-- & -- & -- & --- & -- & -- & -- & -- & -- \\
\hline --- & -- & --- & -- & --- & --- & -- & -- & --- \\
\hline--- & -- & --- & --- & --- & --- & --- & -- & - \\
\hline --- & -- & - & --- & -- & --- & -- & -- & --- \\
\hline$\cdots$ & --- & -- & --- & -- & --- & --- & -- & -- \\
\hline--- & --- & --- & --- & --- & --- & -- & -- & --- \\
\hline- & - & --- & $\ldots$ & --- & --- & -- & -- & -- \\
\hline--- & --- & --- & --- & --- & -- & --- & -- & -- \\
\hline --- & --- & -- & --- & --- & -- & --- & --- & --- \\
\hline-- & --- & --- & --- & --- & --- & -- & --- & --- \\
\hline--- & $\begin{array}{c}2.4,4.6 \\
2.4,6.7 \\
(4 \text { samples) }\end{array}$ & --- & --- & -- & --- & --- & $\begin{array}{cc}0.29, & 0.31 \\
0.31, & 0.28\end{array}$ & --- \\
\hline--- & 8.9 & --- & --- & --- & --- & $\ldots$ & .24 & --- \\
\hline --- & 4.6 &.- & --- & --- & -- & --- & --- & -- \\
\hline
\end{tabular}


Table 3. Summary of bitumen deposits in Arizona

\begin{tabular}{|c|c|c|c|c|c|c|c|c|}
\hline $\begin{array}{l}\text { Map } \\
\text { number }\end{array}$ & Deposit name & County & $\begin{array}{l}\text { Townships } \\
\text { and Ranges }\end{array}$ & Formation & Age & $\begin{array}{l}\text { L1th- } \\
\text { ology }\end{array}$ & $\begin{array}{l}\text { Depth } \\
(\mathrm{ft})\end{array}$ & $\begin{array}{c}\text { Pay } \\
\text { thickness } \\
\text { (ft })\end{array}$ \\
\hline $1 \mathrm{~A}$ & $\begin{array}{l}\text { 1 B1 ack Rock } \\
\text { Canyon }\end{array}$ & Mohave & $\begin{array}{l}\text { T. } 40 \text { N., } \\
\text { R. } 13 \text { W., } \\
\text { secs. 3, } 10 ; \\
\text { T. } 41 \text { N., } \\
\text { R. } 13 \text { W. } \\
\text { secs. } 11,14 \text {, } \\
23,26,35\end{array}$ & Ka1bab & Permian & is & -- & $\rightarrow$ \\
\hline $1 \mathrm{~B}$ & $\begin{array}{l}{ }^{1} \text { Southwest } \\
\text { Black Rock } \\
\text { Canyon }\end{array}$ & $---\mathrm{do}---$ & $\begin{array}{l}\text { T. } 40 \text { N., } \\
\text { R. } 13 \text { W., } \\
\text { secs. } 17,18\end{array}$ & Moenkopi & Triassic & $\operatorname{ss}(?)$ & $-\infty$ & --- \\
\hline 2 & $\begin{array}{l}{ }^{1} \text { Sedona - } \\
\text { Vernon }\end{array}$ & $\begin{array}{l}\text { Coconino, } \\
\text { Navajo, } \\
\text { Apache }\end{array}$ & $\begin{array}{l}\text { T. } 17 \mathrm{~N} \cdot, \\
\text { R. } 6 \mathrm{E} \cdot, \\
\text { to } \mathrm{T} .10 \mathrm{~N} ., \\
\text { R. } 25 \mathrm{E} .\end{array}$ & $\begin{array}{l}\text { Fort Apache } \\
\text { Member of } \\
\text { Supa1 }\end{array}$ & Permian & --- & outcrop & $\begin{array}{l}135 \mathrm{mi} \\
\text { long }\end{array}$ \\
\hline 3 & ${ }^{1}$ St. Johns & Apache & $\begin{array}{l}\text { T. } 13 \mathrm{~N} ., \\
\text { R. } 28 \mathrm{E} .\end{array}$ & Coconino & $--r$ do-n & ss & -- do--- & --- \\
\hline 4 & ${ }^{1}$ Payson & Gila & $\begin{array}{l}\text { T. } 11 \mathrm{~N} ., \\
\text { R. } 10 \mathrm{E} ., \\
\text { sec. } 17\end{array}$ & $\begin{array}{l}\text { Martin } \\
\text { Limestone }\end{array}$ & Devonian & $1 \mathrm{~s}$ & $---\mathrm{do}---$ & --- \\
\hline 5 & $\begin{array}{l}{ }^{1} \text { Dragoon } \\
\text { Mts. - } \\
\text { Tombstone }\end{array}$ & Cochise & --- & $---\mathrm{do}^{---}$ & $---\mathrm{do}_{0}---$ & is & $---d o---$ & -- \\
\hline
\end{tabular}

${ }^{1}$ Ba11 Associates, 1965. 


\begin{tabular}{|c|c|c|c|c|c|c|c|c|}
\hline Acreage & $\begin{array}{c}\text { API } \\
\text { gravity } \\
\text { (degrees) }\end{array}$ & $\begin{array}{l}\text { viscosity } \\
(\mathrm{cp})\end{array}$ & $\begin{array}{l}\text { Porosity } \\
(\%)\end{array}$ & $\begin{array}{l}\text { Permeability } \\
\text { (md) }\end{array}$ & $\begin{array}{l}\text { Water } \\
\text { sat. } \\
(\%)\end{array}$ & $\begin{array}{l}\text { B1tumen } \\
\text { sat. } \\
\text { (\%) }\end{array}$ & $\begin{array}{l}\text { Sulfur } \\
\text { (we \%) }\end{array}$ & $\begin{array}{l}\text { Resource in } \\
\text { place } \\
\text { (m.b.) }\end{array}$ \\
\hline--- & -- & $\overline{---}$ & --- & -- & -- & -- & -- & --- \\
\hline --- & --- & - & -- & --- & -- & -- & -- & $\cdots$ \\
\hline --- & --- & --- & -- & -- & -- & -- & -- & -- \\
\hline-- & -- & -- & --- & --- & --- & $\cdots$ & -- & --- \\
\hline-- & --- & --- & -- & --- & --- & -- & --- & --- \\
\hline-- & -- & $\cdots$ & -- & -- & - & -.. & --- & - \\
\hline
\end{tabular}


Table 4. Summary of bitumen deposits in Arkansas

\begin{tabular}{|c|c|c|c|c|c|c|c|c|}
\hline $\begin{array}{l}\text { Map } \\
\text { number }\end{array}$ & Deposit name & County & $\begin{array}{l}\text { Townships } \\
\text { and Ranges }\end{array}$ & Formation & Age & $\begin{array}{l}\text { Lfth- } \\
\text { ology }\end{array}$ & $\begin{array}{l}\text { Depth } \\
(\mathrm{ft})\end{array}$ & $\begin{array}{c}\text { Pay } \\
\text { thickness } \\
\quad(\mathrm{f} t)\end{array}$ \\
\hline IA & ${ }^{1}$ Delight & Plke & $\begin{array}{l}\text { T. } 8 \text { S., } \\
\text { R. } 24 \text { W. }\end{array}$ & Trinity & $\begin{array}{l}\text { Early } \\
\text { Cretaceous }\end{array}$ & ss & -- & $3-5$ \\
\hline IB & ${ }^{1}$ Pike & ---do--- & $\begin{array}{l}\text { T. } 7 \text { S., } \\
\text { R. } 24 \text { W. }\end{array}$ & $-\cdots$ do-- & $-\cdots$ do-- & ss & outcrop & $24-12$ \\
\hline $1 \mathrm{C}$ & ${ }^{1}$ Murfreesboro & $---\mathrm{do}---$ & $\begin{array}{l}\text { T. } 8 \text { S., } \\
\text { R. } 25 \text { W. }\end{array}$ & $---\mathrm{do} 0--$ & $---\mathrm{do}_{0}--$ & $\begin{array}{l}\text { ss, } \\
\text { grave1 }\end{array}$ & $\rightarrow-\mathrm{do}=-\longrightarrow$ & $--\infty$ \\
\hline 2 & ${ }^{1}$ Lebanon & Sevier & $\begin{array}{l}\text { T. } 8 \text { S., } \\
\text { R. } 30 \text { W., } \\
\text { secs. } 1,11 \text {, } \\
8 ; \text { T. } 8 \text { S., } \\
\text { R. } 29 \text { W., } \\
\text { sec. } 4\end{array}$ & $---\mathrm{do}^{---}$ & $---\mathrm{do}^{---}$ & ss & $---\mathrm{do}^{---}$ & $0.16-1.0$ \\
\hline $3 \mathrm{~A}$ & $1_{\text {F1oss }}$ & Washington & $\begin{array}{l}\text { T. } 13 \mathrm{~N} ., \\
\text { R. } 32 \text { W. }\end{array}$ & Hale & $\begin{array}{l}\text { Pennsyl- } \\
\text { vanian }\end{array}$ & ss & $---\mathrm{do}---$ & - \\
\hline $3 B$ & ${ }^{1}$ Cane H111 & $---d o---$ & $\begin{array}{l}\text { T. } 14 \mathrm{~N} ., \\
\text { R. } 32 \mathrm{~W} .\end{array}$ & $---\mathrm{do}---$ & $---d_{0}---$ & ss & $---\mathrm{do}---$ & --- \\
\hline $4 \mathrm{~A}$ & ${ }^{1}$ Huntsville & Madison & $\begin{array}{l}\text { T. } 16,17 \mathrm{~N} . \\
\text { R. } 26 \mathrm{~W}, \\
\text { sec. } 9\end{array}$ & $--\mathrm{do}---$ & -- do- & ss & --- do--- & --- \\
\hline $4 B$ & $\begin{array}{l}{ }^{1} \text { Southwest } \\
\text { Huntsville }\end{array}$ & -- do--- & $\begin{array}{l}\text { T. } 16 \mathrm{~N} ., \\
\text { R. } 26 \mathrm{~W} . \\
\mathrm{NE} 1 / 4 . \mathrm{SWI} / 4, \\
\text { sec. } 17\end{array}$ & At oka & $---d o---$ & ss & $---d o---$ & -- \\
\hline 5 & ${ }^{1}$ Deer & Newton & $\begin{array}{l}\text { T. } 14 \text { N., } \\
\text { R. } 21 \text { W. }\end{array}$ & Hale & $---d o-\cdots-$ & ss & $---d o---$ & --- \\
\hline 6 & ${ }^{l_{B a t e s v i l l e}}$ & $\begin{array}{l}\text { Indepen- } \\
\text { dence }\end{array}$ & $\begin{array}{l}\text { T. } 13 \mathrm{~N} . \text {, } \\
\text { R. } 6 \mathrm{~W} .\end{array}$ & $\begin{array}{l}\text { Batesville } \\
\text { Sandsone }\end{array}$ & $\begin{array}{l}\text { Mississ- } \\
\text { Ippian }\end{array}$ & ss & $---\mathrm{do}--\rightarrow$ & -- \\
\hline 7 & $\begin{array}{l}1_{\text {Fourche }} \\
\text { Mountain }\end{array}$ & $S \cot t$ & $\begin{array}{l}\text { T. } 1 \text { N., } \\
\text { R. } 31 \text { W. }\end{array}$ & $\begin{array}{l}\text { Jack Fork } \\
\text { Sandstone }\end{array}$ & $\begin{array}{l}\text { Pennsyi- } \\
\text { vanian }\end{array}$ & $\begin{array}{l}\text { ss, } \\
\text { sh }\end{array}$ & --- do $_{0}--$ & -- \\
\hline
\end{tabular}

l Ball Associates, 1965.

${ }^{2}$ Clardy, 1983. 


\begin{tabular}{|c|c|c|c|c|c|c|c|c|}
\hline Acreage & $\begin{array}{c}\text { API } \\
\text { gravity } \\
\text { (degrees) }\end{array}$ & $\begin{array}{l}\text { V1scosity } \\
(\mathrm{cp})\end{array}$ & $\begin{array}{l}\text { Porosity } \\
(\%)\end{array}$ & $\underset{(\mathrm{md})}{\text { Permeabflity }}$ & $\begin{array}{c}\text { Water } \\
\text { sat. } \\
(\%)\end{array}$ & $\begin{array}{c}\text { Bitumen } \\
\text { sat. } \\
(\%)\end{array}$ & $\begin{array}{l}\text { Sulfur } \\
\text { (wt \%) }\end{array}$ & $\begin{array}{l}\text { Resource in } \\
\text { place } \\
\text { (m.b.) }\end{array}$ \\
\hline-- & --- & --- & --- & --- & --- & --- & 17.2 & --- \\
\hline- & - & --- & --- & --- & -- & $\begin{array}{l}5.06,16.53, \\
6.68,8.86, \\
4.58\end{array}$ & -- & -- \\
\hline-- & $\cdots$ & -- & --- & - & $\cdots$ & -- & --- & --- \\
\hline- & -- & - & -- & --- & --- & --- & -- & -- \\
\hline- & $\cdots$ & --- & --- & -- & --- & - & --- & --- \\
\hline-- & --- & --- & --- & --- & --- & "h1gh" & --- & --- \\
\hline-- & --- & $-\cdots$ & -- & --- & --- & --- & -- & -- \\
\hline-- & --- & --- & --- & --- & --- & --- & --- & --- \\
\hline- & -- & --- & -- & -- & - & -- & --- & --- \\
\hline --- & --- & --- & --- & --- & --- & --- & --- & --- \\
\hline --- & -- & --- & -- & -- & -- & -- & --- & --- \\
\hline
\end{tabular}


Table 5. Summary of bitumen deposits in California

\begin{tabular}{|c|c|c|c|c|c|c|c|c|}
\hline $\begin{array}{l}\text { Map } \\
\text { number }\end{array}$ & Deposit name & County & $\begin{array}{l}\text { Townships } \\
\text { and Ranges }\end{array}$ & Formation & Age & $\begin{array}{l}\text { Lfth- } \\
\text { ology }\end{array}$ & $\begin{array}{l}\text { Depth } \\
\text { (ft) }\end{array}$ & $\begin{array}{l}\text { Pay } \\
\text { thickness } \\
\text { (ft) }\end{array}$ \\
\hline 12, & $, 4,5$ Point Arena & Mendocino & $\begin{array}{l}\text { T. } 12 \mathrm{~N} ., \\
\text { R. } 17 \text { W. }\end{array}$ & $\begin{array}{l}\text { Monterey, } \\
\text { Vaqueros } \\
\text { Sandstone }\end{array}$ & Miocene & ss & outcrop & $1-37$ \\
\hline 2 & ${ }^{5}$ Duxburg Pt. & Marin & -- & Monterey & -- do-- & $\begin{array}{l}\text { asphalt } \\
\text { dike }\end{array}$ & --- & -- \\
\hline 3 & $\begin{array}{l}{ }^{6} \text { Santa Cruz } \\
\text { (2 locations) }\end{array}$ & Santa Cruz & $\begin{array}{l}\text { T. } 11 \mathrm{s.}, \\
\text { R. } 3 \text { w., } \\
\text { sec. } 12 ; \\
\text { T. } 11 \mathrm{~S} ., \\
\text { R. } 2 \text { w., } \\
\text { sec. } 5\end{array}$ & $\begin{array}{l}\text { Monterey } \\
\text { Vaqueros } \\
\text { Sandstone }\end{array}$ & $\begin{array}{l}---\mathrm{do}--- \\
---\mathrm{do}---\end{array}$ & ss & $0-100$ & $1-35$ \\
\hline 4 & $\begin{array}{l}{ }^{4} \text { Sargent } \\
\text { Ranch }\end{array}$ & $\begin{array}{l}\text { Santa } \\
\text { Clara }\end{array}$ & $\begin{array}{l}\text { T. } 11 \mathrm{~s} ., \\
\text { R. } 3 \mathrm{E.,} \\
\text { sec. } 36\end{array}$ & $\begin{array}{l}\text { Purisima, } \\
\text { Monterey }\end{array}$ & $\begin{array}{l}\text { P1iocene } \\
\text { Miocene }\end{array}$ & ss & 300 & 75 \\
\hline 5 & 2,5 Chalone $\mathrm{Cr}$. & San Benito & $\begin{array}{l}\text { T. } 17 \text { S., } \\
\text { R. } 8 \text { E., } \\
\text { secs. 32-33 }\end{array}$ & Monterey & $---\mathrm{do}=--$ & $\begin{array}{l}\text { ss, } \\
\text { ark }\end{array}$ & outcrop & 20 \\
\hline 6 & $\begin{array}{l}{ }^{2} \text { Mylar Quarry } \\
\text { (also called) } \\
5 \text { San Lorenzo } \\
\text { Creek }\end{array}$ & $\begin{array}{l}\text { Monterey } \\
\text {---do--- }\end{array}$ & $\begin{array}{l}\text { T. } 19 \text { S., } \\
\text { R. } 9 \text { E., } \\
\text { SE } 1 / 4 \\
\text { sec. } 15\end{array}$ & Monterey & $\begin{array}{l}\text { Pliocene } \\
\text { Miocene }\end{array}$ & $\begin{array}{l}-\mathrm{do}- \\
-\mathrm{do}-\end{array}$ & $\begin{array}{l}-- \text { do--- } \\
-- \text { do--- }\end{array}$ & $\begin{array}{c}30 \\
---d o---\end{array}$ \\
\hline 7 & ${ }^{2} \mathrm{~K}$ ing City & $---\mathrm{do}---$ & $\begin{array}{l}\text { T. } 19 \mathrm{~s} ., \\
\text { R. } 7 \mathrm{E} ., \\
\text { Sl } / 2 \mathrm{sec} .28, \\
\text { NE } 1 / 4 \mathrm{sec} .33 \text {, } \\
\text { W1/2 sec. } 34 ; \\
\text { T. } 20 \mathrm{~S} ., \\
\text { R. } 7 \mathrm{E} ., \\
\text { sec. } 3\end{array}$ & $\begin{array}{l}\text { Santa } \\
\text { Margarita }\end{array}$ & -- do-- & ss & --- do--- & -- \\
\hline 8 & ${ }^{6}$ Paris Valley & ---do--- & $\begin{array}{l}\text { T. } 22 \mathrm{~s} ., \\
\text { R. } 9 \mathrm{E}_{*}, \\
\text { sec. } 4\end{array}$ & Ansberry & -- do--- & ss & --- & $4-84$ \\
\hline
\end{tabular}

\footnotetext{
${ }^{1}$ Adams and Beatty, 1962.

2 Ball Associates, 1965.

${ }^{3}$ California Diviston of 011 and Gas, 1974.

4 de Chadenedes. 1984.

5 Jennings, 1957.

$6_{\text {Lewin and Associates, } 1984 .}$
} 


\begin{tabular}{|c|c|c|c|c|c|c|c|c|c|}
\hline Acreage & $\begin{array}{c}\text { API } \\
\text { gravity } \\
\text { (degrees) }\end{array}$ & $\begin{array}{l}\text { Viscosity } \\
(\mathrm{cp})\end{array}$ & $\begin{array}{l}\text { Porosity } \\
(\%)\end{array}$ & $\begin{array}{l}\text { Pe rmeability } \\
(\pi d)\end{array}$ & $\begin{array}{c}\text { Water } \\
\text { sat. } \\
(\%)\end{array}$ & $\begin{array}{l}\text { Bitumen } \\
\text { sat. } \\
(\%)\end{array}$ & $\begin{array}{l}\text { Sulfur } \\
\text { (wt \%) }\end{array}$ & $\begin{array}{r}\text { Resour } \\
\text { plac } \\
\text { (m.b }\end{array}$ & $\begin{array}{l}\text { ce in } \\
\text { e) }\end{array}$ \\
\hline-- & --- & --- & --- & --- & $\begin{array}{l}26.5 \mathrm{wt} \\
4_{6-13 \mathrm{wt}}\end{array}$ & 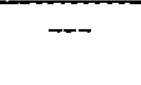 & -- & 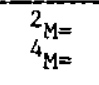 & $\begin{array}{l}1.2 \\
100\end{array}$ \\
\hline --- & -- & -- & -- & --- & --- & --- & -- & \multicolumn{2}{|c|}{$M=\begin{aligned} \text { minor } \\
\text { deposit }\end{aligned}$} \\
\hline --- & $\cdots$ & -- & --- & --- & -- & $10-12$ & -- & $\begin{array}{r}{ }^{6} \mathrm{~S}= \\
\mathrm{T}= \\
{ }^{5} \mathrm{~S}= \\
\mathrm{T}=\end{array}$ & $\begin{array}{l}10 \\
10 \\
20 \\
20\end{array}$ \\
\hline $\begin{array}{l}M=70 \\
T=70\end{array}$ & 10 & 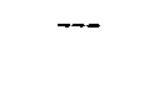 & --- & --- & -- & & ---- & $\mathrm{T}=$ & 400 \\
\hline --- & --- & --. & --- & - & --- & $\underset{w t}{5.7-13.4}$ & --- & & - \\
\hline $\begin{array}{l}1,500^{-} \text {long } \\
100^{-} \text {w1de }\end{array}$ & --- & --- & --- & -- & --- & $\begin{array}{c}8.6-14.4 \\
w t\end{array}$ & --- & & - \\
\hline --- & -- & - & --- & --- & --- & --- & --- & & - \\
\hline --- & --- & $\begin{array}{l}227,000 \\
23,000 \\
87^{\circ} \mathrm{F} \\
(2 \text { lobes })\end{array}$ & 32 & 3,700 & --- & 64 & 1.5 & $\begin{array}{l}S= \\
T=\end{array}$ & $\begin{array}{l}70^{\star} \\
70^{*}\end{array}$ \\
\hline
\end{tabular}


Table 5. Summary of bitumen deposits in California-Continued

\begin{tabular}{|c|c|c|c|c|c|c|c|c|}
\hline $\begin{array}{l}\text { Map } \\
\text { number }\end{array}$ & Deposit name & County & $\begin{array}{l}\text { Townships } \\
\text { and Ranges }\end{array}$ & Formation & Age & $\begin{array}{l}\text { Lith- } \\
\text { ology }\end{array}$ & $\begin{array}{l}\text { Depth } \\
(\mathrm{ft})\end{array}$ & $\begin{array}{c}\text { Pay } \\
\text { thickness } \\
\text { (ft) }\end{array}$ \\
\hline $9 \mathrm{~A}$ & ${ }^{2}$ San Ardo & Monterey & $\begin{array}{l}\text { T. } 22 \text { S., } \\
\text { R. } 9 \text { E., } \\
\text { sec. } 13 \\
\text { T. } 22 \text { S., } \\
\text { R. } 10 \text { E. , } \\
\text { secs. } 18-20, \\
29,32,33\end{array}$ & $\begin{array}{l}\text { Santa } \\
\text { Margarita, } \\
5_{\text {Monterey }}\end{array}$ & $\begin{array}{l}\text { Miocene } \\
--- \text { do--- }\end{array}$ & ss & $\begin{array}{l}5 \mathrm{mi} \\
\text { outcrop }\end{array}$ & 125 \\
\hline $9 B$ & $\begin{array}{l}{ }^{3} \text { Lynch } \\
\text { Canyon } \\
\text { (ollfield) }\end{array}$ & $---d o---$ & $\begin{array}{l}\text { T. } 22 \text { s., } \\
\text { R. } 10 \mathrm{E} . \text {, } \\
\text { sec. } 24\end{array}$ & Monterey & $\begin{array}{l}\text { middle } \\
\text { Miocene }\end{array}$ & --- & 1,800 & 55 \\
\hline 10 & ${ }^{2}$ Bradley & $\rightarrow-\mathrm{do}=--$ & $\begin{array}{l}\text { T. } 24 \text { S., } \\
\text { R. } 10 \text { E., } \\
\text { Swl } / 4 \text { sec. } 35\end{array}$ & $\begin{array}{l}\text { Salinas } \\
\text { Shale }\end{array}$ & $\begin{array}{l}\text { Pliocene, } \\
\text { Miocene }\end{array}$ & ss & outcrop & 30 \\
\hline 12 & $\begin{array}{l}{ }^{6} \text { Edna (Arroyo } \\
\text { Grande) }\end{array}$ & $\begin{array}{l}\text { San Luis } \\
\text { Obispo }\end{array}$ & $\begin{array}{l}\text { T. } 31 \text { S., } \\
\text { R. } 12,13 \mathrm{E} .\end{array}$ & Pismo & Miocene & $\begin{array}{l}\text { ss, } \\
\text { sts, } \\
\text { sh }\end{array}$ & $\begin{array}{l}0-670 \\
0-460 \\
\text { (east } \\
\text { area) }\end{array}$ & -- \\
\hline 13 & $\begin{array}{l}{ }^{3} \text { Huasna } \\
\text { oflfield }\end{array}$ & ---do--- & $\begin{array}{l}\text { T. } 12 \mathrm{~N} ., \\
\text { R. } 33 \mathrm{~W} . \text {, } \\
\text { sec. } 30\end{array}$ & $\begin{array}{l}\text { Santa } \\
\text { Margarita }\end{array}$ & $\begin{array}{l}\text { early } \\
\text { Miocene }\end{array}$ & -- & $750-1560$ & $500-1300$ \\
\hline 14 & $\begin{array}{l}{ }^{3} \text { Guadalupe } \\
\text { oflfield }\end{array}$ & $\begin{array}{l}\text { San Luis } \\
\text { Obispo, } \\
\text { Santa Bar- } \\
\text { bara }\end{array}$ & $\begin{array}{l}\text { T. } 10 \mathrm{~N} ., \\
\text { R. } 36 \mathrm{~W} ., \\
\text { secs. } 1,2, \\
11,12 ; \\
\text { T. } 10 \mathrm{~N} ., \\
\text { R. } 35 \mathrm{W.}, \\
\text { sec. } 6 ; \\
\text { T. } 11 \mathrm{~N} ., \\
\text { R. } 35 \mathrm{~W} ., \\
\text { secs. } 31,32 \text {; } \\
\text { T. } 11 \mathrm{~N} ., \\
\text { R. } 36 \mathrm{~W} ., \\
\text { sec. } 36\end{array}$ & $\begin{array}{l}\text { Monterey- } \\
\text { Point Sal }\end{array}$ & $\begin{array}{l}\text { early } \\
\text { Pliocene } \\
\text { middle } \\
\text { Miocene }\end{array}$ & -- & 3,000 & 200 \\
\hline
\end{tabular}




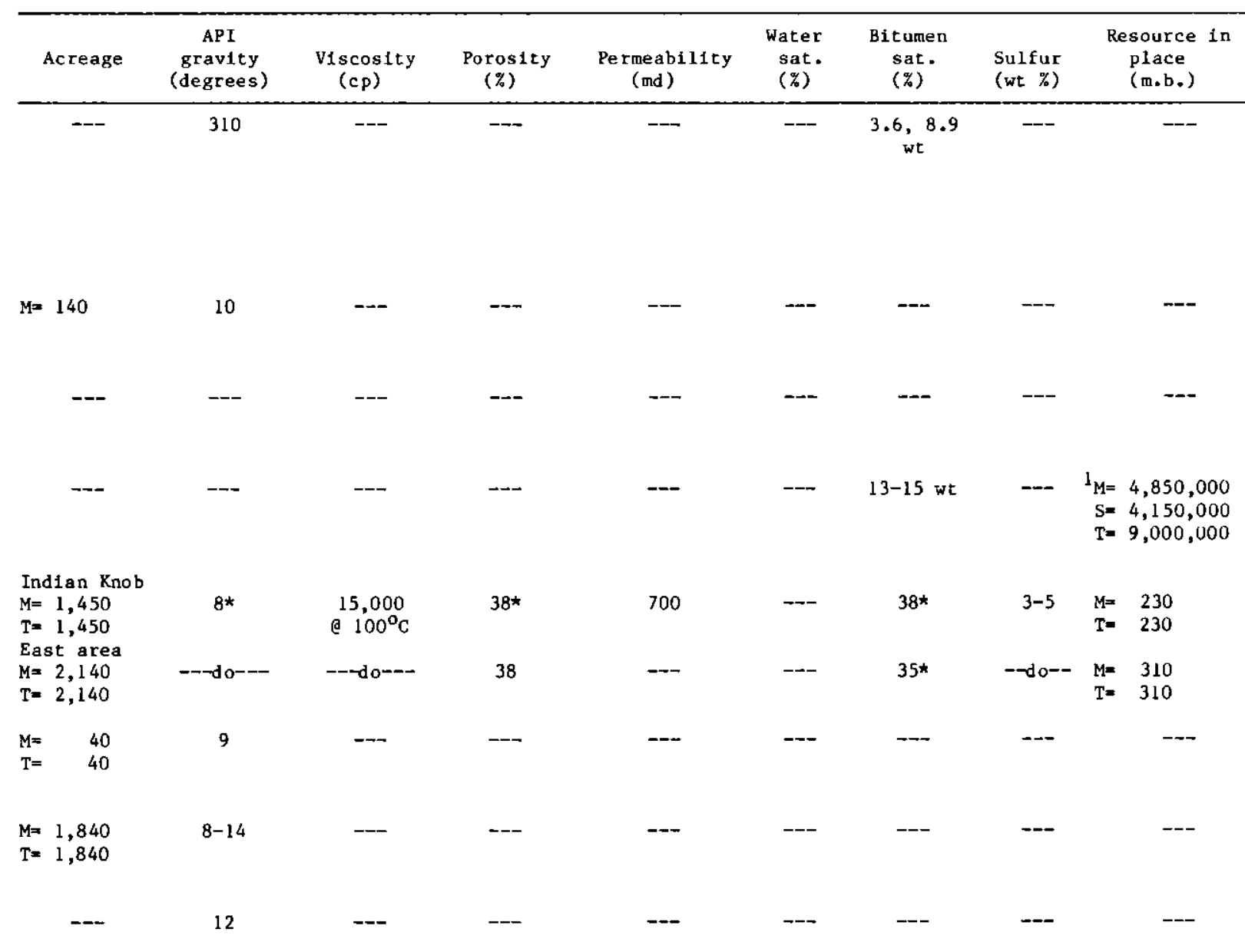


Table 5. Summary of bitumen deposits in California-Continued

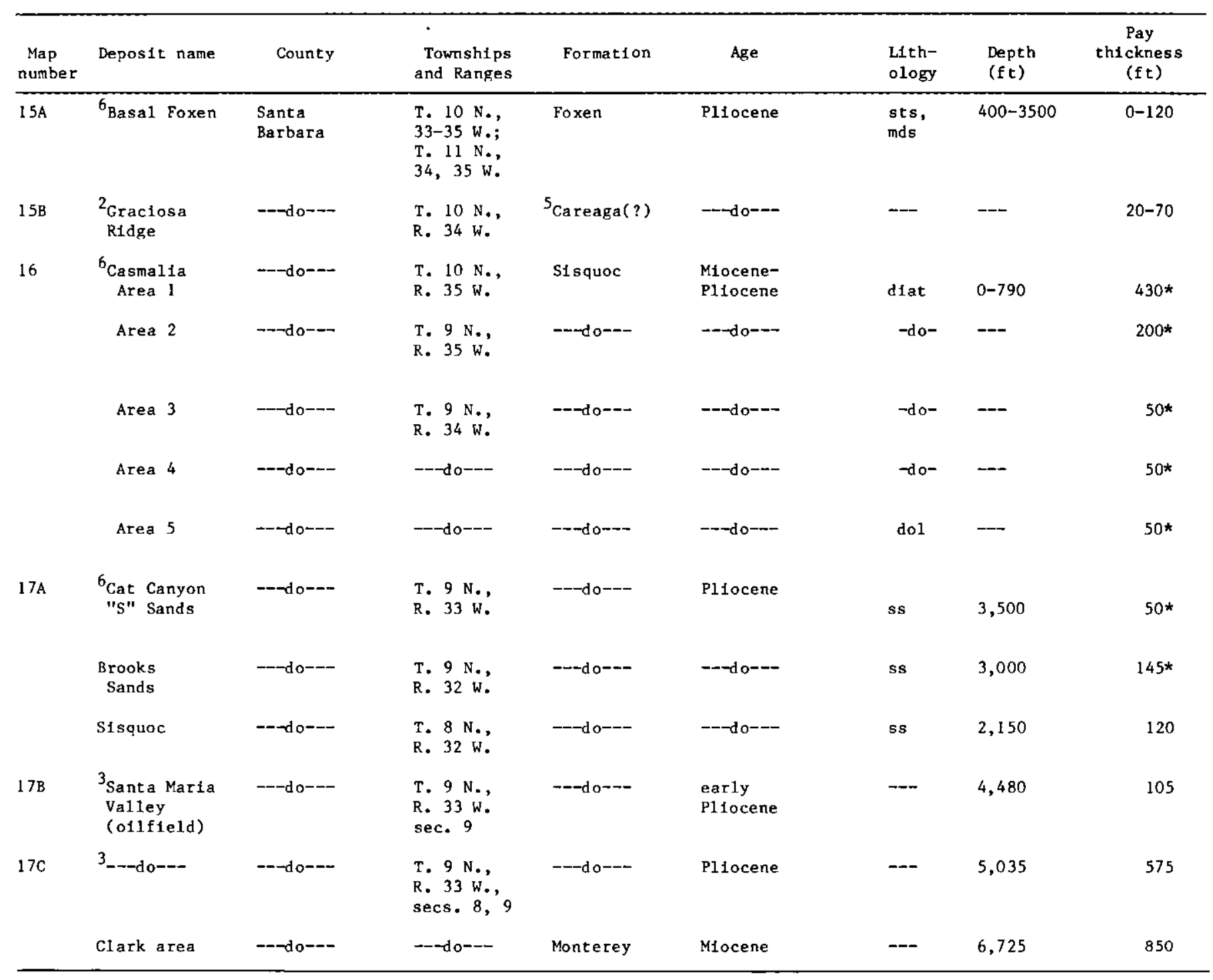




\begin{tabular}{|c|c|c|c|c|c|c|c|c|c|c|}
\hline \multicolumn{2}{|r|}{ Acreage } & $\begin{array}{c}\text { API } \\
\text { gravity } \\
\text { (degrees) }\end{array}$ & $\begin{array}{l}\text { Viscosity } \\
(\mathrm{cp})\end{array}$ & $\begin{array}{l}\text { Porosity } \\
(\%)\end{array}$ & $\begin{array}{l}\text { Permeability } \\
\text { (md) }\end{array}$ & $\begin{array}{l}\text { Water } \\
\text { sat. } \\
(\%)\end{array}$ & $\begin{array}{c}\text { Bitumen } \\
\text { sat. } \\
(\%)\end{array}$ & $\begin{array}{l}\text { Sulfur } \\
\text { (wt \%) }\end{array}$ & $\begin{array}{r}\text { Resourc } \\
\text { place } \\
\text { (m.b. }\end{array}$ & in \\
\hline \multicolumn{2}{|c|}{$\begin{array}{l}\mathrm{S}=25,000 \\
\mathrm{~T}=25,000\end{array}$} & $\begin{array}{l}9-17 \\
9.5 *\end{array}$ & $\begin{array}{l}47,000 \\
\text { e } 80^{\circ} \mathrm{F}\end{array}$ & $25 *$ & $300 *$ & -- & $55 \star$ & $4-5$ & \multicolumn{2}{|c|}{$\begin{array}{l}S=1,900 \\
\mathrm{~T}=1,900\end{array}$} \\
\hline & --- & -- & -- & -- & -- & --- & -- & -- & \multicolumn{2}{|c|}{--} \\
\hline $\begin{array}{l}\mathrm{M}= \\
\mathrm{T}=\end{array}$ & $\begin{array}{l}91 \\
91\end{array}$ & --- & --- & 48 & $<1$ & -- & $31^{*}$ & -- & $\begin{array}{l}M= \\
T=\end{array}$ & $\begin{array}{l}50 \\
50\end{array}$ \\
\hline $\begin{array}{l}\mathrm{M}= \\
\mathrm{S}= \\
\mathrm{T}=\end{array}$ & $\begin{array}{l}115 \\
115 \\
230\end{array}$ & -- & $-\infty$ & 50 & $<1$ & $\cdots$ & 44 & -- & $\begin{array}{l}M= \\
S= \\
T=\end{array}$ & $\begin{array}{l}40 \\
40 \\
80\end{array}$ \\
\hline $\begin{array}{l}S= \\
T=\end{array}$ & $\begin{array}{l}500 \\
500\end{array}$ & --- & --- & --- & --- & --- & --- & --- & $\begin{array}{l}S= \\
T=\end{array}$ & $\begin{array}{l}30 \\
30\end{array}$ \\
\hline $\begin{array}{l}S= \\
T=\end{array}$ & $\begin{array}{l}700 \\
700\end{array}$ & -- & --- & -- & --- & -- & --- & -- & $\begin{array}{l}S= \\
T=\end{array}$ & $\begin{array}{l}40 \\
40\end{array}$ \\
\hline $\begin{array}{l}S= \\
T=\end{array}$ & $\begin{array}{l}1,000 \\
1,000\end{array}$ & - & -- & - & - & - & --- & --- & $\begin{array}{l}\mathrm{S}= \\
\mathrm{T}=\end{array}$ & $\begin{array}{l}60 \\
60\end{array}$ \\
\hline $\begin{array}{l}\mathrm{M}= \\
\mathrm{T}=\end{array}$ & $\begin{array}{l}6,000 \\
6,000\end{array}$ & $6 *$ & $\begin{array}{l}12,000- \\
1,000,000 \\
\text { a res, temp. }\end{array}$ & 32 & $1,400-5,000$ & --- & 52 & --- & $\begin{array}{l}\mathrm{M}= \\
\mathrm{T}=\end{array}$ & $\begin{array}{l}610 \\
610\end{array}$ \\
\hline $\begin{array}{l}\mathrm{M}= \\
\mathrm{T}=\end{array}$ & $\begin{array}{l}740 \\
740\end{array}$ & $\cdots$ & $\begin{array}{l}15,000 \\
\text { res. temp. }\end{array}$ & 37 & 3,450 & --- & 85 & $\longrightarrow$ & $\begin{array}{l}M= \\
T=\end{array}$ & $\begin{array}{l}220 \\
220\end{array}$ \\
\hline $\begin{array}{l}S= \\
T=\end{array}$ & $\begin{array}{l}1,200 \\
1,200\end{array}$ & --- & -- & 31 & $400-1,700$ & --- & 75 & --- & $\begin{array}{l}S= \\
T=\end{array}$ & $\begin{array}{l}280 \\
280\end{array}$ \\
\hline $\begin{array}{l}\mathrm{M}= \\
\mathrm{T}=\end{array}$ & $\begin{array}{l}120 \\
120\end{array}$ & 9 & -- & -- & -- & -- & --- & -- & - & \\
\hline \multirow[t]{2}{*}{$\begin{array}{l}M= \\
T=\end{array}$} & $\begin{array}{l}160 \\
160\end{array}$ & 8 & -- & -- & - & -- & -- & --- & - & \\
\hline & --- & 9 & --- & --- & --- & -- & --- & -- & - & \\
\hline
\end{tabular}


Table 5. Summary of bitumen deposits in California-Continued

\begin{tabular}{|c|c|c|c|c|c|c|c|c|}
\hline $\begin{array}{l}\text { Map } \\
\text { number }\end{array}$ & Deposit name & County & $\begin{array}{l}\text { Townships } \\
\text { and Ranges }\end{array}$ & Formation & Age & $\begin{array}{l}\text { Lith- } \\
\text { ology }\end{array}$ & $\begin{array}{l}\text { Depth } \\
(\mathrm{ft})\end{array}$ & $\begin{array}{c}\text { Pay } \\
\text { th1 ckness } \\
\text { (ft })\end{array}$ \\
\hline \multirow[t]{3}{*}{$17 \mathrm{D}$} & $\begin{array}{l}\text { 6aca-Sisquoc; } \\
\text { Sisquoc- } \\
\text { Laguna } \\
\text { Ranch }\end{array}$ & $\begin{array}{l}\text { Santa } \\
\text { Barbara }\end{array}$ & $\begin{array}{l}\text { T. } 8 \text { N., } \\
\text { R. } 31 \text { W., } \\
\operatorname{secs} .2,3, \\
13\end{array}$ & $\begin{array}{l}\text { Careaga } \\
\text { Sandstone }\end{array}$ & Pliocene & ss & $10-340$ & $100 *$ \\
\hline & $\begin{array}{l}\text { Zaca ofl } \\
\text { fleld }\end{array}$ & $---d o---$ & $\begin{array}{l}\text { T. } 8 \text { N. , } \\
\text { R. } 31 \text { W., } \\
\text { secs. } 29-36\end{array}$ & Monterey & Miocene & $\begin{array}{l}\text { fract. } \\
\text { diat }\end{array}$ & -- & --- \\
\hline & La Brea & ---do--- & $\begin{array}{l}\text { T. } 9 \mathrm{~N}_{.}, \\
\text {R. } 31 \text { W., } \\
\text { sec. } 18\end{array}$ & --- & -- & $\rightarrow$ & --- & -- \\
\hline 18 & $\begin{array}{l}{ }^{2} \text { Sunset- } \\
\text { Maricopa }\end{array}$ & Kern & $\begin{array}{l}\text { T. } 11 \text { N., } \\
\text { R. } 23 \text { W., } \\
\text { secs. } 18-20 ; \\
\text { T. } 11 \text { N., } \\
\text { R. } 24 \text { W.', } \\
\text { secs. 13, } 14\end{array}$ & -- & Miocene & ss & outcrop & $<200-300$ \\
\hline 19 & $\begin{array}{l}{ }^{2} \text { Gaviota } \\
\text { (coasta } 1)\end{array}$ & $\begin{array}{l}\text { Santa } \\
\text { Barbara }\end{array}$ & $\begin{array}{l}\text { T. } 5 \mathrm{~N} . \text {, } \\
\text { R. } 32 \text { W. }\end{array}$ & $\begin{array}{l}{ }^{5} \text { Sisquoc, } \\
\text { Monterey }\end{array}$ & $\begin{array}{l}\text { M1ocene- } \\
\text { P11ocene }\end{array}$ & $\begin{array}{l}\text { cgl } \\
\text { ss }\end{array}$ & outcrop & 25 \\
\hline \multirow[t]{2}{*}{20} & $\begin{array}{l}{ }^{2} \text { Goleta; } \\
\text { La Paterna } \\
\text { Mine }\end{array}$ & ---do--- & $\begin{array}{l}\text { T. } 4 \mathrm{~N} ., \\
\text { R. } 28 \mathrm{~W} .\end{array}$ & Monterey & Miocene & sh & --- & 12 \\
\hline & $\begin{array}{l}\text { More"s } \\
\text { Landing }\end{array}$ & $---d o---$ & ---do--- & -- & $\begin{array}{l}\text { Post- } \\
\text { P1iocene }\end{array}$ & $\begin{array}{l}\text { ss } \\
c l i f f s\end{array}$ & --- & $60-100$ \\
\hline $21 \mathrm{~A}$ & $\begin{array}{l}{ }^{3} \text { Summerland } \\
\text { (oilfield) } \\
\text { onshore area }\end{array}$ & $---d o---$ & $\begin{array}{l}\text { T. } 4 \mathrm{~N} ., \\
\text { R. } 26 \mathrm{~W} ., \\
\text { secs. } 15, \\
16,21,22\end{array}$ & Casitas & $\begin{array}{l}\text { Pleisto- } \\
\text { cene }\end{array}$ & $\rightarrow$ & 140 & 100 \\
\hline $21 \mathrm{~B}$ & $\begin{array}{l}\text { 3---do--- } \\
\text { offshore } \\
\text { area }\end{array}$ & ---do--- & $\begin{array}{l}\text { T. } 4 \mathrm{~N}_{.}, \\
\text {R. } 26 \mathrm{~W}_{.}, \\
\text {sec. } 21\end{array}$ & --- do- & $---d o---$ & -- & 220 & 100 \\
\hline 22 & $\begin{array}{l}{ }^{2} \text { Carpenteria; } \\
\text { Los Conchas } \\
\text { Quarry, } \\
\text { Higgins Mine }\end{array}$ & $---d o---$ & $\begin{array}{l}\text { T. } 4 \text { N., } \\
\text { R. } 25 \text { W. }\end{array}$ & Monterey & Miocene & sand & $10-25$ & $10-15$ \\
\hline $23 \mathrm{~A}$ & ${ }^{2}$ Punta Gorda & Ventura & $\begin{array}{l}\text { T. } 3 \mathrm{~N} . \\
\text { R. } 25 \mathrm{~W} . \\
\text { sec. } 1\end{array}$ & ---do--- & ---do--- & sh & $0-100$ & 0.3 veins \\
\hline $23 \mathrm{~B}$ & $\begin{array}{l}\text { 2Ventura; } \\
\text { La Brea Mine } \\
\text { Canon De1 Diablo }\end{array}$ & $\begin{array}{l}\text { Ventura } \\
0\end{array}$ & $\begin{array}{l}\text { T. } 3 \mathrm{~N}_{.}, \\
\text {R. } 24 \mathrm{~W} \text {. }\end{array}$ & Monterey(?) & Miocene(?) & $\begin{array}{l}\text { cls, } \\
\text { sh }\end{array}$ & --- & $\begin{array}{r}0.60-1.3 \\
\text { veins }\end{array}$ \\
\hline
\end{tabular}




\begin{tabular}{|c|c|c|c|c|c|c|c|c|c|}
\hline & eage & $\begin{array}{c}\text { API } \\
\text { gravity } \\
\text { (degrees) }\end{array}$ & $\begin{array}{l}\text { viscostty } \\
(c p)\end{array}$ & $\begin{array}{l}\text { Porosity } \\
\text { (\%) }\end{array}$ & $\begin{array}{l}\text { Permeability } \\
\text { (md) }\end{array}$ & $\begin{array}{l}\text { Water } \\
\text { sat. } \\
(\%)\end{array}$ & $\begin{array}{l}\text { B1tumen } \\
\text { sat. } \\
(\%)\end{array}$ & $\begin{array}{l}\text { Sulfur } \\
\text { (wt \%) }\end{array}$ & $\begin{array}{l}\text { Resource in } \\
\text { place } \\
\text { (m.b.) }\end{array}$ \\
\hline $\begin{array}{l}M= \\
T=\end{array}$ & $\begin{array}{l}620 \\
620\end{array}$ & -- & -- & $35^{*}$ & --- & --- & $40^{*}$ & --- & $\begin{array}{l}\mathrm{M}=90 \\
\mathrm{~T}=90\end{array}$ \\
\hline \multirow[t]{6}{*}{$\begin{array}{l}M= \\
T=\end{array}$} & $\begin{array}{l}580 \\
580\end{array}$ & $4-6$ & -- & --- & -- & -- & --- & --- & $\begin{array}{l}M=90 \\
T=90\end{array}$ \\
\hline & 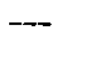 & -- & -- & -- & $\cdots$ & --- & --- & --- & $\begin{array}{ll}S= & 50 \\
T= & 50\end{array}$ \\
\hline & -- & --- & --- & --- & --- & --- & --- & --- & -- \\
\hline & -- & --- & --- & --- & -- & --- & $\begin{array}{c}5_{16-19} \\
\text { gal/ton }\end{array}$ & --- & -- \\
\hline & --- & --- & --- & --- & --- & --- & $16 \mathrm{wt}$ & --- & --- \\
\hline & --- & --- & -- & -- & --- & --- & -- & --- & --- \\
\hline $\begin{array}{l}M= \\
\mathrm{T}=\end{array}$ & $\begin{array}{l}110 \\
110\end{array}$ & 7 & -- & --- & --- & -- & -- & -- & --- \\
\hline \multirow[t]{3}{*}{$\begin{array}{l}M= \\
T=\end{array}$} & $\begin{array}{l}58 \\
58\end{array}$ & 7 & --- & --- & --- & --- & -- & --- & - \\
\hline & -- & -- & --- & --- & --- & - & $19 *$ wt & --- & -- \\
\hline & -- & --- & -- & --- & -- & --- & $\begin{array}{l}28 \\
\text { asphalt }\end{array}$ & --- & --- \\
\hline $\begin{array}{l}M= \\
T=\end{array}$ & $\begin{array}{l}200 \\
200\end{array}$ & --- & -- & -- & --- & - & $\begin{array}{l}40, \\
15-22\end{array}$ & --- & --- \\
\hline
\end{tabular}


Table 5. Summary of bitumen deposits in California-Continued

\begin{tabular}{|c|c|c|c|c|c|c|c|c|}
\hline $\begin{array}{l}\text { Map } \\
\text { number }\end{array}$ & Deposit name & Count $y$ & $\begin{array}{l}\text { Townships } \\
\text { and Ranges }\end{array}$ & Formation & Age & $\begin{array}{l}\text { L1th- } \\
\text { ology }\end{array}$ & $\begin{array}{l}\text { Depth } \\
(\mathrm{ft})\end{array}$ & $\begin{array}{l}\text { Pay } \\
\text { thickness } \\
\text { (ft) }\end{array}$ \\
\hline 24 & ${ }^{2}$ Sulfur Mtn. & Ventura & $\begin{array}{l}\text { T. } 4 \mathrm{~N} ., \\
\text { R. } 21 \mathrm{~W} \text {. }\end{array}$ & Fernando & Ple1stocene & $\begin{array}{l}\text { cgl, } \\
\text { so }\end{array}$ & outcrop & --- \\
\hline $25 \mathrm{~A}$ & ${ }^{6}$ Loma Verde & Los Angeles & $\begin{array}{l}\text { T. } 5 \mathrm{~N}, \\
R_{*} 17 \mathrm{~W}, \\
\text { secs, } 25,26 \text {, } \\
35,36\end{array}$ & $\begin{array}{l}\text { Radovich } \\
\text { Sandstone }\end{array}$ & Pliocene & ss & $\begin{array}{l}500- \\
2,000\end{array}$ & 200 \\
\hline $25 B$ & "Golden" & ---do--- & $\begin{array}{l}\text { T. } 5 \mathrm{~N}_{.}, \\
\text {R. } 17 \mathrm{~W}_{.}, \\
\text {sec. } 35\end{array}$ & Saugus & Ple1stocene & -- & 4,000 & 40 \\
\hline 26 & $2_{\text {Newhall }}$ & ---do--- & $\begin{array}{l}\text { T. } 3 \text { N., } \\
\text { R. } 15 \text { W., } \\
\text { secs. } 7 \text {, } \\
18,19\end{array}$ & $\begin{array}{l}\text { Repetto, } \\
\text { P1co }\end{array}$ & P1tocene & ss & outcrop & -- \\
\hline \multirow[t]{2}{*}{27} & $\begin{array}{l}{ }^{6} \text { oxnard } \\
\text { Vaca Tar } \\
\text { sand }\end{array}$ & Ventura & $\begin{array}{l}\text { T. } 1 \mathrm{~N} . \\
\text { R. } 20-22 \text { W. }\end{array}$ & P1co & $---d o^{--r}$ & ss & $\begin{array}{l}1,800- \\
2,500\end{array}$ & $210^{\star}$ \\
\hline & $\begin{array}{l}\text { Lower Tar } \\
\text { sand }\end{array}$ & ---do--- & $\begin{array}{l}\text { T. } 2 \text { N., } \\
\text { R. } 22 \text { พ. }\end{array}$ & ---do-- & ---do--- & ss & --- & $50 *$ \\
\hline 28 & $\begin{array}{l}{ }^{2} \text { Rancho La } \\
\text { Brea and } \\
\text { L.A. C1ty }\end{array}$ & Los Angeles & $\begin{array}{l}\text { T. } 1 \mathrm{~S} . \text {. } \\
\mathrm{R} .14 \mathrm{~W} \text {. }\end{array}$ & -- & $\begin{array}{l}\text { late } \\
\text { Pleistocene }\end{array}$ & $\begin{array}{l}\text { alluv. } \\
\text { terrace }\end{array}$ & outcrop & -- \\
\hline 29 & $\begin{array}{l}{ }^{3} \text { Gaffey } \\
\text { (odlfleld) }\end{array}$ & ---do--- & $\begin{array}{l}\text { T. } 4 \text { s., } \\
\text { R. } 14 \text { W., } \\
\text { sec. } 35\end{array}$ & Repet to & Pllocene & ss & 1,500 & 100 \\
\hline 30 & ${ }^{2}$ Point Ferman & ---do--- & $\begin{array}{l}\text { T. } 5 \text { S., } \\
\text { R. } 14 \text { W. }\end{array}$ & $\begin{array}{l}\text { Franctscan } \\
\text { Sandstone }\end{array}$ & Jurassic & ss & outcrop & 75 \\
\hline 31 & ${ }^{2}$ Chino & $\begin{array}{l}\text { San } \\
\text { Bernardino }\end{array}$ & $\begin{array}{l}\text { T. } 2 \text { S., } \\
\text { R. } 8 \text { W. }\end{array}$ & -- & $\begin{array}{l}\text { Overlying } \\
\text { Mlocene }\end{array}$ & ss & --- & 20 \\
\hline 32 & Rtehfield & Orange & $\begin{array}{l}\text { T. } 3 \mathrm{~s} ., \\
\text { R. } 9 \text { W., } \\
\text { sec. } 28,32\end{array}$ & Repetto & Pliocene & ss & $\begin{array}{l}2,500- \\
3,200\end{array}$ & $80^{\star}$ \\
\hline $33 \mathrm{~A}$ & Newport Bay & ---do--- & $\begin{array}{l}\text { T. } 6 \mathrm{~S} ., \\
\text { R. } 10 \mathrm{~W} .\end{array}$ & -- & -- & sand & outcrop & --- \\
\hline $33 \mathrm{~B}$ & $\begin{array}{l}\text { Newport } \\
\text { (o11field) }\end{array}$ & Orange & $\begin{array}{l}\text { T. } 6 \mathrm{~S} ., \\
\text { R. } 10 \mathrm{~W} \text {. } \\
\text { sec. } 28\end{array}$ & Puenta & $\begin{array}{l}\text { early } \\
\text { M1ocene }\end{array}$ & -- & 1,500 & 300 \\
\hline
\end{tabular}




\begin{tabular}{|c|c|c|c|c|c|c|c|c|c|c|c|}
\hline & Acreage & $\begin{array}{c}\text { API } \\
\text { gravity } \\
\text { (degrees) }\end{array}$ & $\begin{array}{l}\text { Viscosity } \\
(c)\end{array}$ & $\begin{array}{l}\text { Porosity } \\
(\%)\end{array}$ & $\begin{array}{l}\text { Permeab111ty } \\
\text { (md) }\end{array}$ & $\begin{array}{l}\text { Water } \\
\text { sat. } \\
(\%)\end{array}$ & $\begin{array}{c}\text { Bitumen } \\
\text { sat. } \\
(\%)\end{array}$ & $\begin{array}{l}\text { Sulfur } \\
\text { (wt \%) }\end{array}$ & \multicolumn{3}{|c|}{$\begin{array}{c}\text { Resource in } \\
\text { place } \\
\text { (m.b.) }\end{array}$} \\
\hline & --- & -- & --- & $-\infty$ & $\cdots$ & -- & -- & -- & & --- & \\
\hline \multirow[t]{3}{*}{$\begin{array}{l}S= \\
T=\end{array}$} & $\begin{array}{l}600 \\
600\end{array}$ & --- & --- & 30 & 1,000 & -- & 45 & -- & $\begin{array}{l}\mathrm{S}= \\
\mathrm{T}=\end{array}$ & $\begin{array}{l}90 \\
90\end{array}$ & \\
\hline & -- & 9 & - & -- & $\rightarrow-$ & --- & -- & --- & & -- & \\
\hline & -- & -- & -- & -- & -- & --- & --- & --- & & -- & \\
\hline $\begin{array}{l}\mathrm{M}= \\
\mathrm{T}=\end{array}$ & $\begin{array}{l}1,765 \\
1,765\end{array}$ & 5 & $\begin{array}{l}500,000 \\
\text { @ } 100^{\circ} \mathrm{F}\end{array}$ & 35 & 6,000 & --- & 75 & $6-7$ & $\begin{array}{l}\mathrm{M}= \\
\mathrm{T}=\end{array}$ & $\begin{array}{l}500 \\
500\end{array}$ & \\
\hline \multirow[t]{2}{*}{$\begin{array}{l}S= \\
T=\end{array}$} & $\begin{array}{l}1,900 \\
1,900\end{array}$ & 5 & -- & --- & --- & --- & -- & -- & $\begin{array}{l}S= \\
T=\end{array}$ & $\begin{array}{l}160 \\
160\end{array}$ & \\
\hline & --- & $\cdots$ & 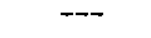 & $\rightarrow$ & $\cdots$ & -- & - & -- & & $\cdots$ & \\
\hline \multirow[t]{5}{*}{$\begin{array}{l}M= \\
T=\end{array}$} & $\begin{array}{l}20 \\
20\end{array}$ & 10 & $-\cdots$ & -- & --- & --- & --- & --- & & -- & \\
\hline & -- & --- & --- & --- & -- & --- & -- & --- & & --- & \\
\hline & -- & --- & -- & --- & -- & --- & -- & --- & & --- & \\
\hline & -- & $3_{12}$ & - & --- & -- & -- & -- & -- & $\begin{array}{l}\mathrm{S}= \\
\mathrm{T}=\end{array}$ & $\begin{array}{l}40 \\
40\end{array}$ & \\
\hline & -- & --- & -- & --- & --- & --- & --- & --- & & --- & \\
\hline$M=$ & 25 & 9 & -- & -- & -- & -- & --- & --- & & -- & \\
\hline
\end{tabular}


Table 6. Summary of bitumen deposits in Colorado

\begin{tabular}{|c|c|c|c|c|c|c|c|c|}
\hline $\begin{array}{l}\text { Map } \\
\text { number }\end{array}$ & Deposit name & County & $\begin{array}{l}\text { Townships } \\
\text { and Ranges }\end{array}$ & Formation & Age & $\begin{array}{l}\text { Lith- } \\
\text { ology }\end{array}$ & $\begin{array}{r}\text { Depth } \\
\text { (ft) }\end{array}$ & $\begin{array}{l}\text { Pay } \\
\text { th1ckness } \\
\text { (ft) }\end{array}$ \\
\hline 1 & $\begin{array}{l}1_{\text {White River }} \\
\text { Plateau }\end{array}$ & Garfield & $\begin{array}{l}\text { T. } 4,5 \text { s., } \\
\text { R. } 91-93 \text { W. }\end{array}$ & $\begin{array}{l}\text { Maroon, } \\
\text { Weber }\end{array}$ & Permi an & ss & outcrop & 43 \\
\hline 2 & $\begin{array}{l}\text { lPetrolite } \\
\text { Hills }\end{array}$ & Rio $\mathrm{Bl}$ anco & $\begin{array}{l}\text { T. } 2 \text { S., } \\
\text { R. } 94 \text { W. }\end{array}$ & $\begin{array}{l}\text { Douglas Creek } \\
\text { Member of } \\
\text { Green River }\end{array}$ & Eocene & ss & $\begin{array}{l}\text { scattered } \\
\text { outcrops }\end{array}$ & $6-8 *$ \\
\hline 3 & ${ }^{1}$ Gray H111s & $\begin{array}{l}\text { Moffat, } \\
\text { R1o Blanco }\end{array}$ & $\begin{array}{l}\text { T. } 2,3 \mathrm{~N} ., \\
\text { R. } 95-96 \text { W. }\end{array}$ & Wasatch & $\begin{array}{l}\text { late } \\
\text { Eocene }\end{array}$ & ss & $---\mathrm{do}---$ & $0-60$ \\
\hline 4 & $\begin{array}{l}1_{\text {Blue }} \\
\text { Mountain } \\
\text { Plateau }\end{array}$ & Moffat & $\begin{array}{l}\text { T. } 4,5 \mathrm{~N} . \\
\text { R. } 100- \\
103 \mathrm{~W} . ; \\
\text { T. } 6 \mathrm{~N} . \\
\text { R. } 102 \mathrm{~W} \text {. }\end{array}$ & $\begin{array}{l}\text { Weber, } \\
\text { Sh1narump, } \\
\text { Navajo, } \\
\text { Entrada }\end{array}$ & $\begin{array}{l}\text { Permian, } \\
\text { Pennsylvanian, } \\
\text { Trtassic, } \\
\text { Jurassic }\end{array}$ & ss & $---d o---$ & -- \\
\hline
\end{tabular}

l Ball Associates, 1965.

Table 7. Summary of bitumen deposits in Kentucky

\begin{tabular}{|c|c|c|c|c|c|c|c|c|}
\hline $\begin{array}{l}\text { Map } \\
\text { number }\end{array}$ & Deposit name & County & $\begin{array}{l}\text { Carter } \\
\text { coordinates }\end{array}$ & Formation & Age & $\begin{array}{l}\text { Ldth- } \\
\text { ology }\end{array}$ & $\begin{array}{l}\text { Depth } \\
\text { (ft) }\end{array}$ & $\begin{array}{c}\text { Pay } \\
\text { thickness } \\
\text { (ft) }\end{array}$ \\
\hline${ }_{2 \mathrm{~A}}^{1 \mathrm{~A}} 2$, & ${ }^{3} \mathrm{Big}$ Clifty & $\begin{array}{l}\text { Edmonson, } \\
\text { Warren, Logan, } \\
\text { Butler, } \\
\text { Grayson, } \\
\text { Breckinridge }\end{array}$ & $\begin{array}{l}\mathrm{F}-31-\mathrm{K}-41 \\
\text { minor } 1 \mathrm{n} \\
\mathrm{M}-34,35 \\
\mathrm{~N}-34,35\end{array}$ & Big C1ifty & $\begin{array}{l}\text { Mississ- } \\
\text { Ippian }\end{array}$ & ss & $0-600$ & $10-25 *$ \\
\hline $1 \mathrm{~B}$ & ${ }^{2}$ Hardinsburg & $---\mathrm{do}---$ & $\begin{array}{l}F-33-F-40 \\
M-35, N-35\end{array}$ & Hardinsburg & 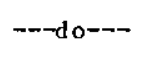 & ss & $125-440$ & $12^{\star}$ \\
\hline $\begin{array}{l}1 \mathrm{C} \\
2 \mathrm{C}\end{array}$ & $\begin{array}{l}{ }^{2} \text { Tar Springs } \\
\text { (2 areas) }\end{array}$ & $\begin{array}{l}\text { Breck1nridge, } \\
\text { Butler }\end{array}$ & $\begin{array}{l}\mathrm{H}, \mathrm{N}-\mathrm{O}, \\
33-36\end{array}$ & $\begin{array}{l}\text { Tar Springs } \\
\text { Member of } \\
\text { Lietchfield }\end{array}$ & $---\mathrm{d} o^{-}--$ & ss & outcrop & $25^{*}$ \\
\hline $1 \mathrm{D}$ & ${ }^{2}$ Caseyville & $\begin{array}{l}\text { Edmonson, } \\
\text { Grayson }\end{array}$ & $\begin{array}{l}\mathrm{K}-\mathrm{H}, \\
36-40\end{array}$ & Caseyville & $\begin{array}{l}\text { Pennsy1- } \\
\text { vanian }\end{array}$ & ss & $20-170$ & $5-40$ \\
\hline 3 & $\begin{array}{l}{ }^{1} \text { Summit, } \\
\text { Solway }\end{array}$ & Hardin & -- & Big Clifty & $\begin{array}{l}\text { Mississ- } \\
\text { Ippian }\end{array}$ & -- & -- & 6 \\
\hline 4 & ${ }^{1}$ Garffeld & Breckinridge & $\begin{array}{l}2 \mathrm{mi} \text {. south } \\
\text { of Garfield }\end{array}$ & --- do--- & $---\mathrm{do}---$ & --- & $10-20$ & 14 \\
\hline 5 & $1_{\text {Beech Grove }}$ & McLean & --- & Caseyville(?) & $\begin{array}{l}\text { Pennsyl- } \\
\text { vanion }\end{array}$ & -- & -- & -- \\
\hline 6 & ${ }^{1}$ Cedar Creek & Ne1son & -- & $\begin{array}{l}\text { "Cornifer- } \\
\text { ous" (prob. } \\
\text { Onondaga) }\end{array}$ & Devontan & is & --- & 6 \\
\hline 7 & ${ }^{1}$ Ravenna & Estill & -- & ---do--- & $---d o--$ & ss & 130 & -- \\
\hline 8 & $\begin{array}{l}{ }^{2} \text { Palntsville } \\
\text { quadrangle }\end{array}$ & $\begin{array}{l}\text { Johnson, } \\
\text { Morgan }\end{array}$ & -- & $\begin{array}{l}\text { Lee } \\
\text { Sandstone }\end{array}$ & $\begin{array}{l}\text { Pennsy1- } \\
\text { vanian }\end{array}$ & -- & $1_{60}$ & $1_{4-12}$ \\
\hline 9 & ${ }^{2}$ Soldier & Carter, Rowan & --- & ---do--- & $--\mathrm{do}---$ & ss & $15-20$ & $5-10$ \\
\hline
\end{tabular}




\begin{tabular}{|c|c|c|c|c|c|c|c|c|}
\hline Acreage & $\begin{array}{c}\text { API } \\
\text { gravity } \\
\text { (degrees) }\end{array}$ & $\begin{array}{l}\text { Viscosity } \\
(c p)\end{array}$ & $\begin{array}{l}\text { Porosity } \\
(\%)\end{array}$ & $\begin{array}{l}\text { Permeability } \\
\text { (md) }\end{array}$ & $\begin{array}{l}\text { Water } \\
\text { sat. } \\
(\%)\end{array}$ & $\begin{array}{l}\text { Bitumen } \\
\text { sat. } \\
\text { (\%) }\end{array}$ & $\begin{array}{l}\text { Sulfur } \\
\text { (wt z) }\end{array}$ & $\begin{array}{l}\text { Resource in } \\
\text { place } \\
\text { (m.b.) }\end{array}$ \\
\hline--- & -- & --- & --- & -- & -- & --- & -- & -- \\
\hline--- & --- & --- & -- & --- & --- & "low" & --- & --- \\
\hline-- & --- & -- & --- & -- & -- & --- & --- & -- \\
\hline-- & -- & -- & $\rightarrow$ & - & --- & --- & --- & -- \\
\hline
\end{tabular}

\begin{tabular}{|c|c|c|c|c|c|c|c|c|c|c|}
\hline \multicolumn{2}{|r|}{ Acreage } & \multirow{2}{*}{$\frac{\begin{array}{c}\text { API } \\
\text { gravity } \\
\text { (degrees) }\end{array}}{10}$} & \multirow{2}{*}{$\begin{array}{l}\text { Viscosity } \\
(c p)\end{array}$} & \multirow{2}{*}{$\begin{array}{c}\text { Porosity } \\
(\%)\end{array}$} & \multirow{2}{*}{$\frac{\begin{array}{c}\text { Permeability } \\
\text { (md) }\end{array}}{100}$} & \multirow{2}{*}{$\begin{array}{c}\text { Water } \\
\text { sat. } \\
(\%)\end{array}$} & \multirow{2}{*}{$\frac{\begin{array}{c}\text { Bitumen } \\
\text { sat. } \\
(\%)\end{array}}{1_{6-7}^{45}}$} & \multirow{2}{*}{$\begin{array}{c}\begin{array}{c}\text { Sulfur } \\
(w t \%)\end{array} \\
--\end{array}$} & \multirow{2}{*}{\multicolumn{2}{|c|}{ 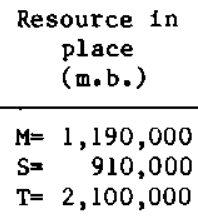 }} \\
\hline $\begin{array}{l}\mathrm{M}= \\
\mathrm{S}= \\
\mathrm{T}=\end{array}$ & $\begin{array}{l}150,000 \\
200,000 \\
350,000\end{array}$ & & & & & & & & & \\
\hline $\begin{array}{r}4 M= \\
S= \\
T=\end{array}$ & $\begin{array}{r}66,000 \\
88,000 \\
154,000\end{array}$ & ${ }^{4} 10$ & --- & 14 & $10-400$ & --- & $40 *$ & --- & $\begin{array}{r}{ }^{4} M= \\
S= \\
T=\end{array}$ & $\begin{array}{l}250,000 \\
180,000 \\
430,000\end{array}$ \\
\hline $\begin{array}{l}\mathrm{S}= \\
\mathrm{T}=\end{array}$ & $\begin{array}{l}34,000 \\
34,000\end{array}$ & $-\infty$ & -- & 20 & $10-100$ & --- & $21^{*}$ & -- & $\begin{array}{l}S= \\
T=\end{array}$ & $\begin{array}{l}340,000 \\
340,000\end{array}$ \\
\hline $\begin{array}{l}M= \\
S= \\
T=\end{array}$ & $\begin{array}{l}35,000 \\
44,000 \\
79,000\end{array}$ & --- & --- & ${ }^{3} 19.2 *$ & $>100$ & --- & $\begin{array}{l}0-60 \\
341 *\end{array}$ & -- & $\begin{array}{l}M= \\
S= \\
T=\end{array}$ & $\begin{array}{l}300,000 \\
250,000 \\
550,000\end{array}$ \\
\hline & --- & --- & --- & $3_{22.7 *}$ & ${ }^{3} 160$ & --- & $\begin{array}{c}7-8 \text { wt } \\
39 *\end{array}$ & -- & & -- \\
\hline & --- & -- & --- & --- & --- & -- & $4-8$ wt & --- & & --- \\
\hline & --- & -- & - & --- & $-\infty$ & --- & -- & --- & & -- \\
\hline & --- & --- & --- & --- & --- & -- & -- & --- & & -- \\
\hline & - & - & -- & --- & --- & -- & 25 vol. & --- & & --- \\
\hline & --- & --- & --- & --- & -- & -- & $4-10 \mathrm{wt}$ & --- & & -- \\
\hline $3_{T}=$ & $<3 \mathrm{ml} d \mathrm{la}$. & --- & -- & -- & --- & --- & $<3$ & -- & & --- \\
\hline
\end{tabular}


Table 8. Summary of bitumen deposits in Michigan

\begin{tabular}{|c|c|c|c|c|c|c|c|c|c|}
\hline $\begin{array}{c}\text { Map } \\
\text { number }\end{array}$ & Deposit & name & County & Location & Formation & Age & $\begin{array}{l}\text { Lith- } \\
\text { ology }\end{array}$ & $\begin{array}{l}\text { Depth } \\
(\mathrm{ft})\end{array}$ & $\begin{array}{c}\text { Pay } \\
\text { thickness } \\
(\mathrm{ft})\end{array}$ \\
\hline 1 & ${ }^{1}$ Rapid $R$ & i ver & Delta & $\begin{array}{l}\text { Near Rapld } \\
\text { River }\end{array}$ & $\begin{array}{l}\text { Trenton } \\
\text { Limestone }\end{array}$ & Ordovician & $1 \mathrm{~s}$ & outcrop & 300 \\
\hline
\end{tabular}

$1_{\text {Ball Assoctates, } 1965 .}$

Table. 9. Summary of bitumen deposits in Mississippi

\begin{tabular}{|c|c|c|c|c|c|c|c|c|}
\hline $\begin{array}{l}\text { Map } \\
\text { number }\end{array}$ & Deposit name & County & $\begin{array}{l}\text { Townships } \\
\text { and Ranges }\end{array}$ & Formation & Age & $\begin{array}{l}\text { Lith- } \\
\text { ology }\end{array}$ & $\begin{array}{l}\text { Depth } \\
\quad(f t)\end{array}$ & $\begin{array}{r}\text { Pay } \\
\text { thickness } \\
(\mathrm{ft})\end{array}$ \\
\hline IA & ${ }^{1}$ Cypress Pond & Tishomingo & $\begin{array}{l}\text { T. } 5 \mathrm{~S} ., \\
\mathrm{R} \cdot 11 \mathrm{E} \cdot, \\
\text { sec. } 17,18\end{array}$ & $\begin{array}{l}\text { Bangor } \\
\text { Limestone }\end{array}$ & $\begin{array}{l}\text { Mississ- } \\
\text { ippian }\end{array}$ & ls & outcrop & -- \\
\hline lB & $1_{\text {Margerum West }}$ & $---\mathrm{do} 0--$ & $\begin{array}{l}\text { T. } 4 \text { S., } \\
\text { R. } 11 \mathrm{E} \cdot, \\
\text { sec. } 22,27\end{array}$ & --- do-r- & --- do--- & $\begin{array}{l}\text { ls, } \\
\text { sh }\end{array}$ & $---\mathrm{do}_{\mathrm{o}}---$ & -- \\
\hline $1 \mathrm{C}$ & $\begin{array}{l}{ }^{1} \text { Southward's } \\
\text { Ford }\end{array}$ & --- do--- & $\begin{array}{l}\text { T. } 5 \text { S., } \\
\text { R. } 11 \mathrm{E} .\end{array}$ & $\begin{array}{l}\text { Hartselle } \\
\text { Sandstone }\end{array}$ & $\longrightarrow-\longrightarrow$ do-- & ss & $---\mathrm{do}---$ & $-\infty$ \\
\hline
\end{tabular}

lBall Assoclates, 1965.

Table 10. Summary of bitumen deposits in Montana

\begin{tabular}{|c|c|c|c|c|c|c|c|c|}
\hline $\begin{array}{l}\text { Map } \\
\text { number }\end{array}$ & Deposit name & County & $\begin{array}{l}\text { Townships } \\
\text { and Ranges }\end{array}$ & Formation & Age & $\begin{array}{l}\text { Lith- } \\
\text { ology }\end{array}$ & $\begin{array}{l}\text { Depth } \\
(\mathrm{ft})\end{array}$ & $\begin{array}{c}\text { Pay } \\
\text { thickness } \\
\text { (ft) }\end{array}$ \\
\hline 1 & $\begin{array}{l}{ }^{1} \text { Dupuyer-Sun } \\
\text { River }\end{array}$ & Teton & $\begin{array}{l}\text { T. } 27 \mathrm{~N} \cdot, \\
\text { R. } 8 \mathrm{~W} .- \\
\text { T. } 22 \mathrm{~N}, \\
\text { R. } 8 \mathrm{~W} .\end{array}$ & $\begin{array}{l}\text { Colorado } \\
\text { Shale }\end{array}$ & Cretaceous & $\begin{array}{l}\text { sh, } \\
1 \mathrm{~s}\end{array}$ & outcrop & 12 \\
\hline 2 & $\begin{array}{l}{ }^{1} \text { Butcher } \\
\text { Creek }\end{array}$ & Carbon & $\begin{array}{l}\text { T. } 6 \mathrm{~S} ., \\
\text { R. } 18 \mathrm{E} \cdot, \\
\text { sec. } 32\end{array}$ & Fort Union & Paleocene & $\begin{array}{l}\text { ss, } \\
\text { sh }\end{array}$ & outcrop & -- \\
\hline 3 & $1_{\text {Red Dome }}$ & $\rightarrow--\mathrm{do}_{0}--\overline{1}$ & $\begin{array}{l}\text { T. } 7 \text { s., } \\
\text { R. } 24 \text { E., } \\
\text { sec. } 17, \\
18,20\end{array}$ & Chugwater & Triassic & $\operatorname{sh}(?)$ & outcrop & $20-27$ \\
\hline
\end{tabular}

$\mathrm{l}_{\text {Ball Associates, } 1965 .}$ 


\begin{tabular}{|c|c|c|c|c|c|c|c|c|}
\hline Acreage & $\begin{array}{c}\text { API } \\
\text { gravity } \\
\text { (degrees) }\end{array}$ & $\begin{array}{l}\text { Viscosity } \\
(c p)\end{array}$ & $\begin{array}{l}\text { Porosity } \\
(\%)\end{array}$ & $\begin{array}{l}\text { Permeability } \\
\text { (md) }\end{array}$ & $\begin{array}{c}\text { Water } \\
\text { sat. } \\
(\%)\end{array}$ & $\begin{array}{c}\text { Bitumen } \\
\text { sat. } \\
(\%)\end{array}$ & $\begin{array}{l}\text { Sulfur } \\
\text { (wt \%) }\end{array}$ & $\begin{array}{l}\text { Resource in } \\
\text { place } \\
\text { (m.b.) }\end{array}$ \\
\hline--- & --- & -- & -- & --- & --- & --- & --- & - \\
\hline
\end{tabular}

\begin{tabular}{|c|c|c|c|c|c|c|c|c|}
\hline Acreage & $\begin{array}{c}\text { API } \\
\text { gravity } \\
\text { (degrees) }\end{array}$ & $\begin{array}{l}\text { Viscosity } \\
\text { (cp) }\end{array}$ & $\begin{array}{l}\text { Porosity } \\
(\%)\end{array}$ & $\begin{array}{l}\text { Permeability } \\
\text { (md) }\end{array}$ & $\begin{array}{c}\text { Water } \\
\text { sat. } \\
(\%)\end{array}$ & $\begin{array}{c}\text { Bitumen } \\
\text { sat. } \\
(\%)\end{array}$ & $\begin{array}{l}\text { Sulfur } \\
\text { (wt \%) }\end{array}$ & $\begin{array}{l}\text { Resource in } \\
\text { place } \\
\text { (m.b.) }\end{array}$ \\
\hline$\rightarrow$ & -- & -- & 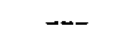 & --- & --- & - & - & --- \\
\hline--- & -- & -- & --- & -- & $=$ & -- & $\cdots$ & $-\cdots$ \\
\hline$-\infty$ & --- & --- & --- & --- & --- & --- & $-\infty$ & --- \\
\hline
\end{tabular}

\begin{tabular}{|c|c|c|c|c|c|c|c|c|}
\hline Acreage & $\begin{array}{c}\text { API } \\
\text { gravity } \\
\text { (degrees) }\end{array}$ & $\begin{array}{l}\text { Viscosity } \\
(c p)\end{array}$ & $\begin{array}{l}\text { Porosity } \\
(\%)\end{array}$ & $\begin{array}{l}\text { Permeability } \\
\text { (md) }\end{array}$ & $\begin{array}{c}\text { Water } \\
\text { sat. } \\
(\%)\end{array}$ & $\begin{array}{l}\text { B1tumen } \\
\text { sat. } \\
(\%)\end{array}$ & $\begin{array}{l}\text { Sulfur } \\
\text { (wt. \%) }\end{array}$ & $\begin{array}{c}\text { Resource in } \\
\text { place } \\
\text { (m.b.) }\end{array}$ \\
\hline-- & --- & --- & -- & $-\infty$ & $-\infty$ & 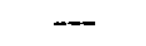 & --- & --- \\
\hline$-\sim-$ & --- & --- & --- & -- & --- & --- & --- & --- \\
\hline-- & -- & -- & --- & --- & $\rightarrow$ & $2.45-6.60$ & - & $--\infty$ \\
\hline
\end{tabular}


Table 11. Summary of bitumen deposits in New Mexico

\begin{tabular}{|c|c|c|c|c|c|c|c|c|}
\hline $\begin{array}{c}\text { Map } \\
\text { number }\end{array}$ & Deposit name & County & $\begin{array}{l}\text { Townships } \\
\text { and Ranges }\end{array}$ & Formation & Age & $\begin{array}{l}\text { Lith- } \\
\text { ology }\end{array}$ & $\begin{array}{l}\text { Depth } \\
(\mathrm{ft})\end{array}$ & $\begin{array}{c}\text { Pay } \\
\text { thickness } \\
\text { (ft) }\end{array}$ \\
\hline 1 & ${ }^{2}$ Santa Rosa & Guadalupe & $\begin{array}{l}\text { T. } 9,10 \mathrm{~N} . \\
\text { R. } 21,22 \mathrm{E}\end{array}$ & Santa Rosa & $\operatorname{Tr} 1 \operatorname{ass} 1 \mathrm{c}$ & ss & $\begin{array}{c}\text { outcrop } \\
\text { to } 170 \\
20^{\star}\end{array}$ & $38^{\star}$ \\
\hline 2 & $\begin{array}{l}{ }^{1} \text { Ga1lup } \\
{ }^{3} \text { (P1 nedale) }\end{array}$ & McKInley & $\begin{array}{l}\text { T. } 16 \mathrm{N.}, \\
\text { R. } 16 \mathrm{~W} ., \\
\text { sec. } 11\end{array}$ & $\begin{array}{l}\text { Gallup } \\
3 \text { Member of } \\
\text { Torrivio } \\
\text { Sandstone }\end{array}$ & Cretaceous & ss & outcrop & $\begin{array}{r}3 \\
328\end{array}$ \\
\hline
\end{tabular}

$l_{\text {Ball Associates, } 1965 .}$

2 Lewin and Associates, 1984.

3 Molenaar, 1977.

Table 12. Summaray of bitumen deposits in New York

\begin{tabular}{|c|c|c|c|c|c|c|c|c|}
\hline $\begin{array}{l}\text { Map } \\
\text { number }\end{array}$ & Deposit name & County & $\begin{array}{l}\text { Townships } \\
\text { and Ranges }\end{array}$ & Formation & Age & $\begin{array}{l}\text { Lith- } \\
\text { ology }\end{array}$ & $\begin{array}{l}\text { Depth } \\
(\mathbf{f t})\end{array}$ & $\begin{array}{c}\text { Pay } \\
\text { thickness } \\
\text { (ft) }\end{array}$ \\
\hline 1 & ${ }^{1}$ Rochester & Monroe & Western N.Y. & Ni agra Group & Silurian & ls & outcrop & --- \\
\hline 2 & $1_{\text {Rockville }}$ & Allegany & Rockville & Chemung Group & Devonian & ss & $-\mathrm{de}-\mathrm{C}$ & - \\
\hline 3 & $1_{\text {Laona }}$ & Chaut auqua & $\begin{array}{l}2 \text { mi-SE. of } \\
\text { Fredonia }\end{array}$ & $\begin{array}{l}\text { Portage } \\
\text { Group }\end{array}$ & $\begin{array}{l}\text { Late } \\
\text { Devonian }\end{array}$ & ss & $---d o---$ & 5 \\
\hline
\end{tabular}

${ }_{\text {Ball Associates, } 1965 .}$

Table 13. Summary of bitumen deposits in Ohio

\begin{tabular}{|c|c|c|c|c|c|c|c|c|}
\hline $\begin{array}{l}\text { Map } \\
\text { number }\end{array}$ & Deposit name & County & $\begin{array}{l}\text { Townships } \\
\text { and Ranges }\end{array}$ & Formation & Age & $\begin{array}{l}\text { Lith- } \\
\text { ology }\end{array}$ & $\begin{array}{l}\text { Depth } \\
(\mathbf{f t})\end{array}$ & $\begin{array}{l}\text { Pay } \\
\text { hickness } \\
(\mathrm{ft})\end{array}$ \\
\hline $1 \mathrm{~A}$ & $\mathrm{l}_{\mathrm{Fa} 11 \mathrm{sv} 111 \mathrm{e}}$ & Highland & $\begin{array}{l}11 / 2 \text { mi. S. } \\
\text { of Carey town }\end{array}$ & $\begin{array}{l}\text { Pebbles Member } \\
\text { Nlagra Series }\end{array}$ & $\begin{array}{l}\text { Late } \\
\text { Silurian }\end{array}$ & dol & $\begin{array}{l}\text { exposed } \\
\text { In quarry }\end{array}$ & 10 \\
\hline IB & $1_{\mathrm{H} 111 \mathrm{sboro}}$ & $---\mathrm{do}---$ & $\begin{array}{l}2.5 \mathrm{mi} . \mathrm{NW} \\
\text { of } \mathrm{HIllsboro}\end{array}$ & $\begin{array}{l}\text { Li } 11 \text { y Member } \\
\text { Niagra Series }\end{array}$ & Silurian & do 1 & $---\mathrm{do}^{-}--$ & 20 \\
\hline $1 \mathrm{C}$ & ${ }^{1}$ Brick School & $---\mathrm{d} O---$ & $\begin{array}{l}5 \mathrm{mi} . \mathrm{NW} \text { of } \\
\text { Hi11s boro }\end{array}$ & $---\mathrm{do}---$ & $---\mathrm{d} 0^{---}$ & dol & "Mod. & 1. thick" \\
\hline $2 \mathrm{~A}$ & $1_{\text {Locust Grove }}$ & Ad ams & $\begin{array}{l}1 / 4 \mathrm{ml} \cdot \mathrm{SE} \\
\text { Locust Grove }\end{array}$ & $\begin{array}{l}\text { Pebbles Member } \\
\text { Niagra Series }\end{array}$ & $\begin{array}{l}\text { Late } \\
\text { S1lurian }\end{array}$ & dol & $\begin{array}{l}\text { exposed } \\
\text { in quarry }\end{array}$ & 22 \\
\hline $2 \mathrm{~B}$ & $\begin{array}{l}\text { lsinking }_{\text {Spring }} \\
\text { Sing }\end{array}$ & Highland & $\begin{array}{l}1.5 \mathrm{mi} . \mathrm{N} \\
\text { of Sinking } \\
\text { Spring }\end{array}$ & ---do--- & $---\mathrm{do}---$ & dol & -- dor-- & 3 \\
\hline 3 & $1_{\text {Buckland }}$ & Auglaize & $\begin{array}{l}\text { T } 4 \mathrm{~S} . \\
\text { R. } 5 \mathrm{E} \text {. }\end{array}$ & $\begin{array}{l}\text { Waterlime } \\
\text { or Helderburg }\end{array}$ & Devonian & Is & outcrop & -- \\
\hline
\end{tabular}

${ }^{1}$ Ball Associates, 1965. 


\begin{tabular}{|c|c|c|c|c|c|c|c|c|c|}
\hline & Acreage & $\begin{array}{c}\text { API } \\
\text { gravity } \\
\text { (degrees) }\end{array}$ & $\begin{array}{l}\text { Viscosity } \\
(c p)\end{array}$ & $\begin{array}{l}\text { Porosity } \\
(\%)\end{array}$ & $\begin{array}{l}\text { Permeability } \\
\text { (md) }\end{array}$ & $\begin{array}{c}\text { Water } \\
\text { sat. } \\
(\%)\end{array}$ & $\begin{array}{l}\text { Bitumen } \\
\text { sat. } \\
(\%)\end{array}$ & $\begin{array}{l}\text { Sulfur } \\
\text { (wt \%) }\end{array}$ & $\begin{array}{c}\text { Resource in } \\
\text { place } \\
\left(\mathrm{m} \cdot \mathrm{b}_{.}\right)\end{array}$ \\
\hline \multirow[t]{2}{*}{$\begin{array}{l}M= \\
S= \\
T=\end{array}$} & $\begin{array}{r}6,100 \\
12,000 \\
18,100\end{array}$ & 12 & $\begin{array}{l}30,000 \\
\text { (i. } 60^{\circ} \mathrm{F}\end{array}$ & $10 *$ & 150 & --- & $5 *$ & 2.2 & $\begin{array}{l}M=130,000 \\
S=220,000 \\
T=350,000\end{array}$ \\
\hline & -- & -- & $\rightarrow--$ & --- & --- & --- & --- & & $-\infty$ \\
\hline
\end{tabular}

\begin{tabular}{|c|c|c|c|c|c|c|c|c|}
\hline Acreage & $\begin{array}{c}\text { API } \\
\text { gravity } \\
\text { (degrees) }\end{array}$ & $\begin{array}{l}\text { viscos } 1 t y \\
(c p)\end{array}$ & $\begin{array}{l}\text { Porosity } \\
(\%)\end{array}$ & $\begin{array}{l}\text { Permeabi11ty } \\
\text { (md) }\end{array}$ & $\begin{array}{l}\text { Water } \\
\text { sat. } \\
(\%)\end{array}$ & $\begin{array}{l}\text { Bitumen } \\
\text { sat. } \\
(\%)\end{array}$ & $\begin{array}{l}\text { Sulfur } \\
\text { (wt \%) }\end{array}$ & $\begin{array}{l}\text { Resource in } \\
\text { place } \\
\text { (m.b.) }\end{array}$ \\
\hline -די & -- & -- & --- & -- & -- & -- & -- & $-\cdots$ \\
\hline-- & $-\infty$ & --- & -- & --- & --- & "high" & $-\infty$ & --- \\
\hline-- & --- & --- & --- & --- & -- & $>2$ & -- & -- \\
\hline
\end{tabular}

\begin{tabular}{|c|c|c|c|c|c|c|c|c|}
\hline Acreage & $\begin{array}{c}\text { API } \\
\text { gravity } \\
\text { (degrees) }\end{array}$ & $\begin{array}{l}\text { V1 scosity } \\
(c p)\end{array}$ & $\begin{array}{c}\text { Porosity } \\
(\%)\end{array}$ & $\begin{array}{l}\text { Permeability } \\
\text { (md) }\end{array}$ & $\begin{array}{c}\text { Water } \\
\text { sat. } \\
(\%)\end{array}$ & $\begin{array}{c}\text { Bitumen } \\
\text { sat. } \\
(\%)\end{array}$ & $\begin{array}{l}\text { Sulfur } \\
(w t \%)\end{array}$ & $\begin{array}{c}\text { Resource in } \\
\text { place } \\
\text { (m.b.) }\end{array}$ \\
\hline - & -- & --- & --- & -- & -- & $<2$ & -- & --- \\
\hline$=-$ & --- & -- & - & $\cdots$ & --- & 1.5 & --- & -- \\
\hline$-\infty$ & --- & $-\infty$ & -- & $-\infty$ & --- & 2.5 & --- & --- \\
\hline$-\infty$ & -- & --- & --- & --- & $=-r$ & $<1$ & $-\infty$ & --- \\
\hline--- & --- & -- & - & --- & -- & $<1$ & -- & -- \\
\hline - & - & $-\infty$ & $-\sim$ & -- & $m$ & -- & $-\infty$ & ---- \\
\hline
\end{tabular}


Table 14. Summary of bitumen deposits in Oklahoma

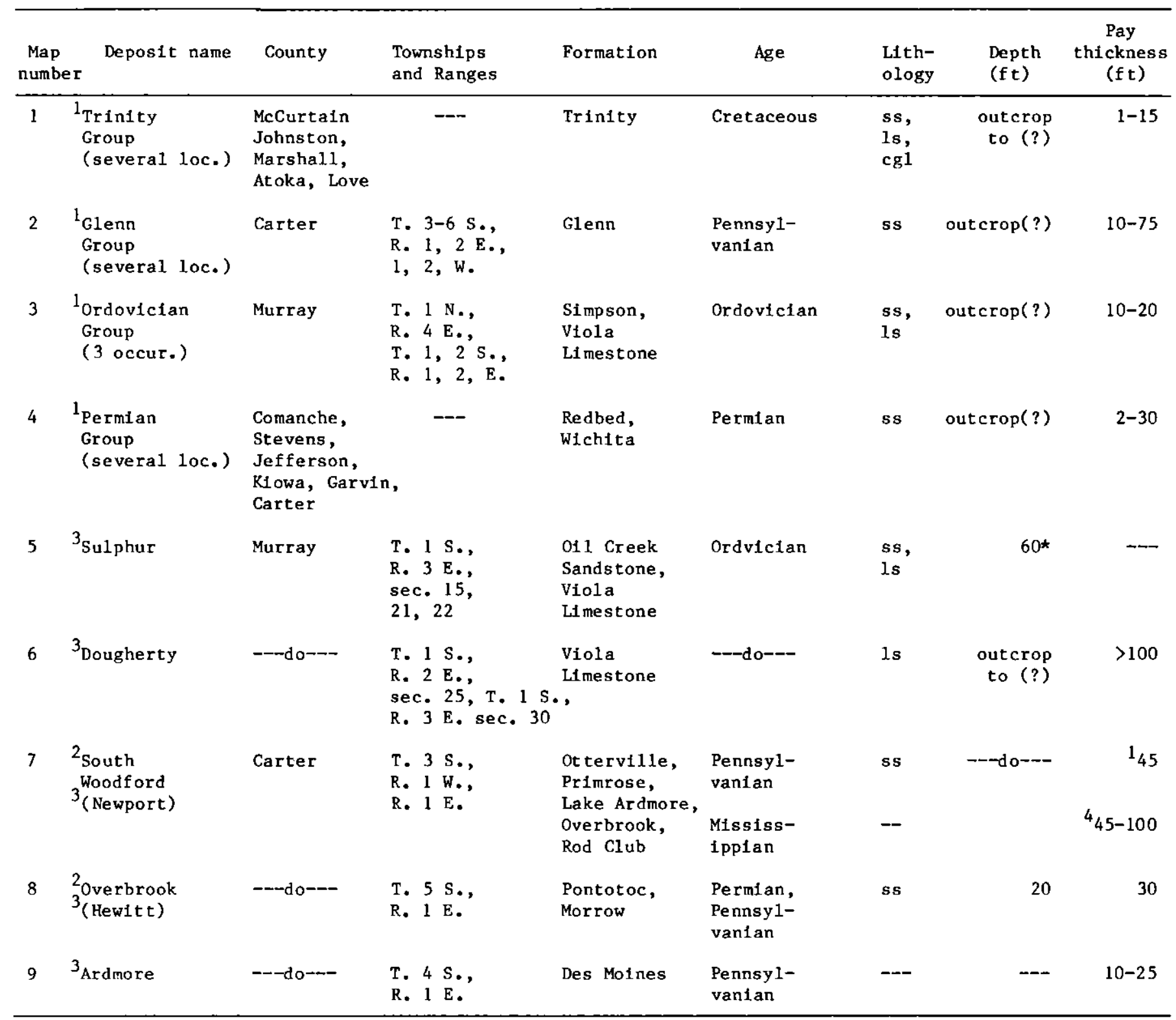

1 Ball Assoctates, 1965.

2 Harrison and Burchfield, 1984.

${ }^{3}$ Lewin and Associates, 1984.

${ }_{5}^{4}$ Harrison, et al, 1981 .

5 Williams, 1982. 


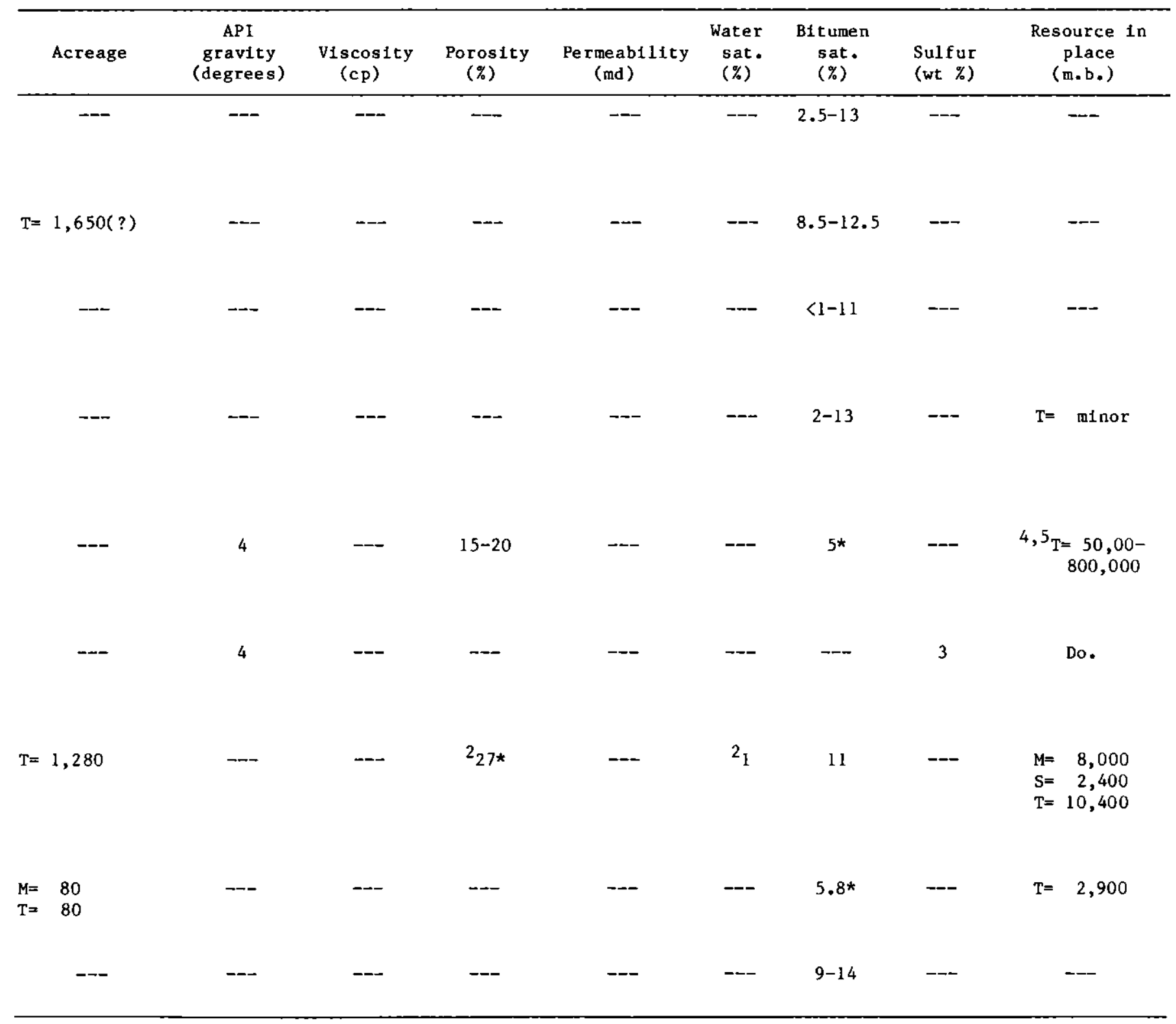


Table 15. Summary of bitumen deposits in Texas

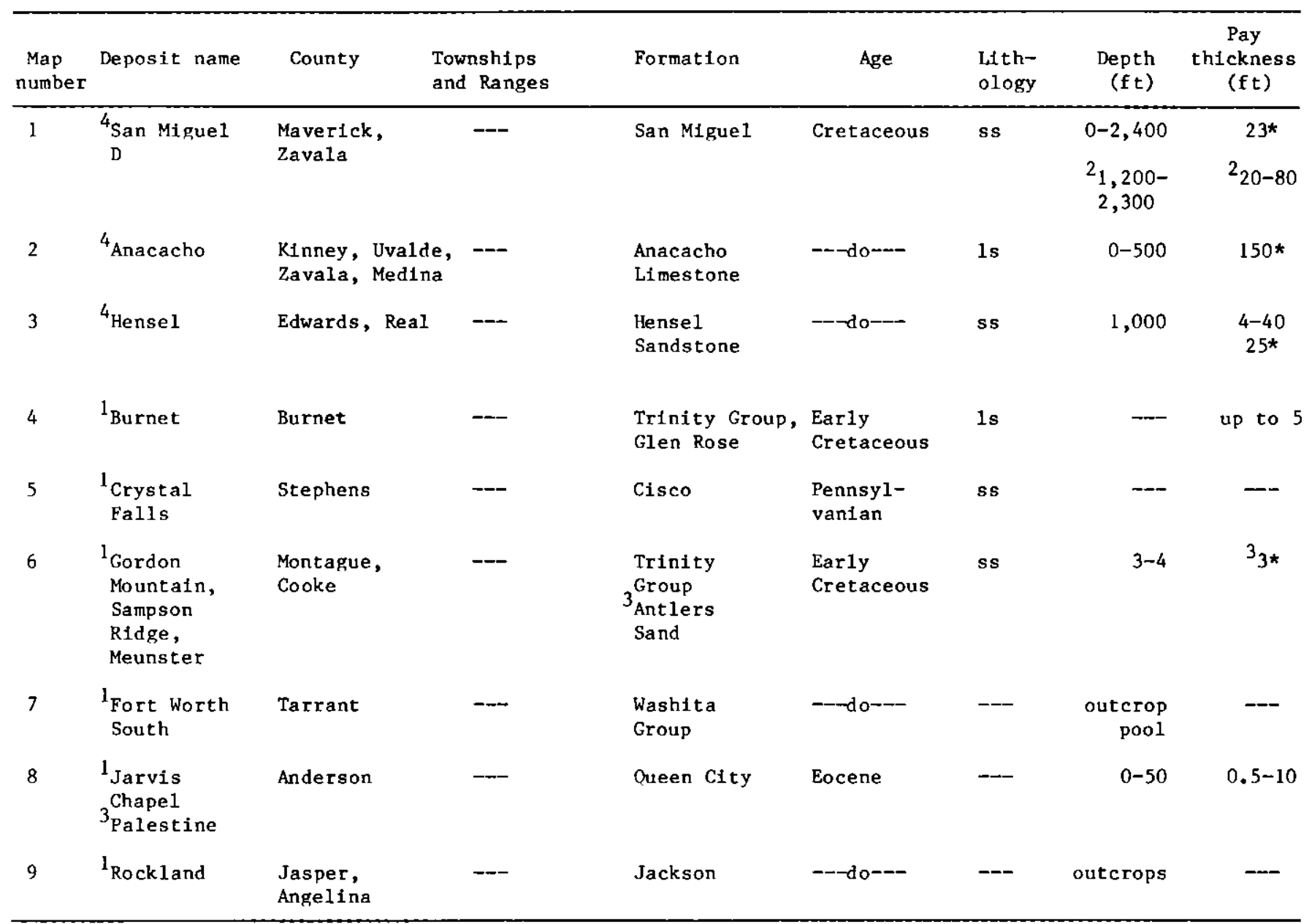

\footnotetext{
$\mathrm{I}_{\text {Ball Assoclates, } 1965 .}$

2 Britton, 1984 .

3 Evans, 1975.

${ }^{4}$ Lewin and Associates, 1984.
} 


\begin{tabular}{|c|c|c|c|c|c|c|c|c|c|c|}
\hline \multicolumn{2}{|c|}{ Acreage } & \multirow{2}{*}{$\frac{\begin{array}{c}\text { API } \\
\text { gravity } \\
\text { (degrees) }\end{array}}{(-2)-10}$} & $\begin{array}{l}\text { Viscosity } \\
\text { (cp) }\end{array}$ & \multirow{2}{*}{$\begin{array}{c}\begin{array}{c}\text { Porosity } \\
(\%)\end{array} \\
26-30\end{array}$} & \multirow{2}{*}{$\begin{array}{c}\begin{array}{c}\text { Permeability } \\
\text { (md) }\end{array} \\
\begin{array}{c}50-100+ \\
2250-1,000\end{array}\end{array}$} & \multirow{2}{*}{$\begin{array}{c}\text { Water } \\
\text { sat. } \\
(\%)\end{array}$} & \multirow{2}{*}{$\begin{array}{c}\begin{array}{c}\text { B1tumen } \\
\text { sat. } \\
(\%)\end{array} \\
40^{\star}\end{array}$} & \multirow{2}{*}{$\frac{\begin{array}{c}\text { Sulfur } \\
\text { (wt \%) }\end{array}}{10}$} & \multicolumn{2}{|c|}{$\begin{array}{c}\text { Resource in } \\
\text { place } \\
\text { (m.b.) }\end{array}$} \\
\hline $\begin{array}{l}M= \\
S= \\
T=\end{array}$ & $\begin{array}{r}115,000 \\
49,000 \\
164,000\end{array}$ & & $\begin{array}{c}20,000- \\
20,000,000 \\
\text { res. temp. }\end{array}$ & & & & & & $\begin{array}{l}\mathrm{M}= \\
\mathrm{S}= \\
\mathrm{T}=\end{array}$ & $\begin{array}{r}3,200,000 \\
610,000 \\
3,810,000\end{array}$ \\
\hline $\begin{array}{l}M= \\
S= \\
T=\end{array}$ & $\begin{array}{r}8,400 \\
20,000 \\
28,400\end{array}$ & -- & $\begin{array}{c}235,000 \\
70^{\circ} \mathrm{F} \\
200 @ 150^{\circ} \mathrm{F}\end{array}$ & 25 & $0.2-7,000$ & -- & 50 & 3 & $\begin{array}{l}M= \\
S= \\
T=\end{array}$ & $\begin{array}{l}550,000 \\
400,000 \\
950,000\end{array}$ \\
\hline \multirow[t]{5}{*}{$\begin{array}{l}M= \\
T=\end{array}$} & $\begin{array}{l}8,100 \\
8,100\end{array}$ & 7 & $\begin{array}{l}450 @ 210^{\circ} \mathrm{F} \\
75 \text { @ } 277^{\circ} \mathrm{F}\end{array}$ & 29 & 8,400 & -- & 52 & 5 & $\begin{array}{l}M= \\
M=\end{array}$ & $\begin{array}{l}120,000 \\
120,000\end{array}$ \\
\hline & -- & --- & --- & - & --- & --- & $34.4 \mathrm{wt}$ & -- & $3_{\mathrm{T}}=$ & 11 \\
\hline & -- & --- & --- & --- & -- & --- & -- & --- & & --- \\
\hline & -- & --- & - & -- & --- & --- & $10 \mathrm{wt}$ & -- & & --- \\
\hline & -- & -- & --- & --- & $-\infty$ & --- & -- & --- & & --- \\
\hline \multicolumn{11}{|c|}{3 deposits } \\
\hline & $\begin{array}{l}198 \\
198\end{array}$ & --- & - & -- & --- & --- & $\begin{array}{l}\text { trace } \\
\text { to full }\end{array}$ & - & ${ }^{3} \mathrm{~T}=$ & 550 \\
\hline & --- & -- & --- & -- & --- & -- & $3>27$ & --- & & -- \\
\hline
\end{tabular}


Table 16. Summary of bitumen deposits in Utah

\begin{tabular}{|c|c|c|c|c|c|c|c|c|}
\hline $\begin{array}{l}\text { Map } \\
\text { number }\end{array}$ & Deposit name & County & $\begin{array}{l}\text { Townships } \\
\text { and Ranges }\end{array}$ & Formation & Age & $\begin{array}{l}\text { Lith- } \\
\text { ology }\end{array}$ & $\begin{array}{l}\text { Depth } \\
\text { (ft) }\end{array}$ & $\begin{array}{l}\text { Pay } \\
\text { thickness } \\
\text { (ft) }\end{array}$ \\
\hline lB & ${ }^{5}$ Gould Ranch & --- do--- & $\begin{array}{l}\text { T.42 S., } \\
\text { R. } 13 \text { W. }\end{array}$ & $---d o---$ & $---d o---$ & $1 \mathrm{~s}$ & $\rightarrow-\mathrm{do}^{--}$ & --- \\
\hline 1C & $\begin{array}{l}{ }^{5} \text { Hurricane } \\
\text { Cliffs }\end{array}$ & $---\mathrm{d} 0-\cdots$ & $---d_{0}---$ & $---\mathrm{do}--$ & $---d o---$ & ls & $---d o^{---}$ & --- \\
\hline $1 \mathrm{D}$ & ${ }^{5}$ North Creek & $---\mathrm{do}_{0}--$ & $\begin{array}{l}\text { T. } 41 \mathrm{~S} ., \\
\text { R. } 11 \mathrm{~W} .\end{array}$ & Moenkopi & $---\mathrm{do}---$ & sh & $---\mathrm{do}---$ & --- \\
\hline 2 & $\begin{array}{l}{ }^{2} \text { Circle Cliffs } \\
\text { (East Flank, } \\
\text { West Flank; }\end{array}$ & Garfield & $\begin{array}{l}{ }^{5} \text { T. } 33-36 \\
\text { S., R. 6-9 } \\
\text { E. }\end{array}$ & $---\mathrm{do}^{-}--$ & $---d o---$ & $\begin{array}{l}\text { ss, } \\
\text { sts }\end{array}$ & $\begin{array}{r}50-400 \\
\text { some } \\
\text { outcrops }\end{array}$ & $5_{5-300}$ \\
\hline 3 & $\begin{array}{l}{ }^{4} \text { White Canyon } \\
\text { Flat }\end{array}$ & $---d o---$ & $\begin{array}{l}\text { T. } 33 \mathrm{~s} ., \\
\mathrm{R} .7 \mathrm{E} .\end{array}$ & Chinle & --do--- & ss & $5_{0-220}$ & $5_{5-21}$ \\
\hline 4 & ${ }^{4}$ Mexican Hat & San Juan & $\begin{array}{l}\text { T. } 41-42 \mathrm{~S} . \\
\text { R. } 17-19 \mathrm{E} .\end{array}$ & $\begin{array}{l}\text { Rico, } \\
\text { Hermosa }\end{array}$ & $\begin{array}{l}\text { Pennsylvantan- } \\
\text { Permian, } \\
\text { Pennsylvanian }\end{array}$ & $\begin{array}{l}1 \text { ss } \\
1 s\end{array}$ & -- & --- \\
\hline 5 & ${ }^{4}$ White Canyon & $---\mathrm{do}$ & $\begin{array}{l}\text { T. } 34-35 \mathrm{~S} ., \\
\text { R. } 15,16 \mathrm{E} .\end{array}$ & $\begin{array}{l}\text { Moenkopi, } \\
\text { Cutler }\end{array}$ & $\begin{array}{l}\text { Triassic, } \\
\text { Permian }\end{array}$ & $1_{\text {ss }}$ & --- & -- \\
\hline $6 \mathrm{~B}$ & $\begin{array}{l}5 \text { Tar Sand } \\
\text { Triangle } \\
\text { (unnamed minor } \\
\text { occurrences) }\end{array}$ & Garfleld & $\begin{array}{l}\text { T. } 32 \text { l/2, } \\
33 \mathrm{~S} ., \mathrm{R} .14, \\
15 \mathrm{E} .\end{array}$ & $\begin{array}{l}\text { Wh1te R1m } \\
\text { Member of } \\
\text { Cutler }\end{array}$ & Permian & ss & -- & - \\
\hline
\end{tabular}

${ }^{1}$ Campbe11 and Ritzma, 1979.

${ }_{3}^{2}$ Lewin and Associates, 1984.

3 Peterson and Ritzma, 1974.

${ }_{5}^{4}$ Ritzma, 1968.

5 Rit zma, 1979 . 


\begin{tabular}{|c|c|c|c|c|c|c|c|c|c|}
\hline Acreage & $\begin{array}{c}\text { API } \\
\text { gravity } \\
\text { (degrees) }\end{array}$ & $\begin{array}{l}\text { Viscosity } \\
(c p)\end{array}$ & $\begin{array}{l}\text { Porosity } \\
(\%)\end{array}$ & $\begin{array}{l}\text { Permeability } \\
\quad \text { (md) }\end{array}$ & $\begin{array}{c}\text { Water } \\
\text { sat. } \\
(\%)\end{array}$ & $\begin{array}{l}\text { B1tumen } \\
\text { sat. } \\
(\%)\end{array}$ & $\begin{array}{l}\text { Sulfur } \\
\text { (wt \%) }\end{array}$ & & $\begin{array}{c}\text { Resource in } \\
\text { place } \\
\text { (m.b.) }\end{array}$ \\
\hline--- & -- & -- & --- & -- & -- & -- & -- & $\mathrm{T}=$ & $500-1,000$ \\
\hline-- & - & -- & -- & --- & --- & --- & -- & & --- \\
\hline-- & -- & -- & -- & --- & --- & --- & -- & & --- \\
\hline-- & - & -- & -- & -- & --- & - & --- & & -- \\
\hline $\begin{array}{lr}M= & 6,400 \\
S= & 14,000 \\
T= & 20,400\end{array}$ & ${ }^{1}(-7)-2$ & --- & $13-17$ & 80 & --- & --- & $\begin{array}{l}1_{3.6} \\
5_{3.96}\end{array}$ & $\begin{array}{l}M= \\
S= \\
T=\end{array}$ & $\begin{array}{r}560,000 \\
1,140,000 \\
1,700,000\end{array}$ \\
\hline--- & --- & -- & --- & -- & -- & -- & $5_{3.5}$ & $\begin{array}{r}5_{\mathrm{M}}= \\
\mathrm{S}= \\
\mathrm{T}=\end{array}$ & $\begin{array}{l}1,800 \\
1,000 \\
2,800\end{array}$ \\
\hline-- & -- & --- & -- & --- & --- & --- & --- & $\mathrm{T}=$ & $400-500$ \\
\hline--- & --- & --- & --- & $\ldots$ & -- & -- & $5_{2.7}$ & $5_{T=}$ & $\begin{array}{l}12,000- \\
15,000\end{array}$ \\
\hline $\begin{aligned} 2_{M} & =93,000 \\
S & =55,000 \\
T & =148,000\end{aligned}$ & $14.3 *$ & $\ldots$ & $\begin{array}{c}222^{*} \\
\text { White R1m } \\
17 \star \\
\text { Moenkop1 }\end{array}$ & $268 *$ & 14.7 & $2_{40}$ & $1_{3.8 *}$ & $\begin{array}{l}\mathrm{M}= \\
\mathrm{S}= \\
\mathrm{T}=\end{array}$ & $\begin{array}{c}2,300,000 \\
10,200,000- \\
13,700,000 \\
12,500,000- \\
16,000,000\end{array}$ \\
\hline-- & --- & --- & -- & --- & -- & --- & --- & $\begin{array}{l}M= \\
S= \\
T=\end{array}$ & $\begin{array}{r}500 \\
2,500 \\
3,000\end{array}$ \\
\hline
\end{tabular}


Table 16. Summary of bitumen deposits in Utah-Continued

\begin{tabular}{|c|c|c|c|c|c|c|c|c|}
\hline $\begin{array}{l}\text { Map } \\
\text { number }\end{array}$ & Deposit name & County & $\begin{array}{l}\text { Townships } \\
\text { and Ranges }\end{array}$ & Formation & Age & $\begin{array}{l}\text { Lith- } \\
\text { ology }\end{array}$ & $\begin{array}{l}\text { Depth } \\
\text { (ft) }\end{array}$ & $\begin{array}{l}\text { Pay } \\
\text { thickness } \\
\quad(\mathrm{ft})\end{array}$ \\
\hline $6 C$ & $\begin{array}{l}{ }^{5} \text { Poison Spring } \\
\text { Canyon (near } \\
\text { Tar Sand Tri- } \\
\text { angle) }\end{array}$ & Garfield & $\begin{array}{l}\text { T. } 31 \text { S. , } \\
\text { R. } 13,14 \mathrm{E} .\end{array}$ & Moenkop1 & Triassic & ss & $0-500+$ & $5-24$ \\
\hline 7 & $\begin{array}{l}{ }^{4} \text { Teasdale } \\
{ }^{1} \text { Capitol Reef } \\
\text { (several loc.) }\end{array}$ & Wayne & $\begin{array}{l}\text { T. } 29,30 \mathrm{~s} . \\
\mathrm{R} .6,7, \mathrm{E} .\end{array}$ & $\begin{array}{l}\text { Kaibab, } \\
\text { Moenkop1 }\end{array}$ & $\begin{array}{l}\text { Permian, } \\
\text { Triassic }\end{array}$ & $\begin{array}{l}\text { 1s, } \\
\text { ss }\end{array}$ & -- & --- \\
\hline 8 & $\begin{array}{l}5 \text { Thousand Lake } \\
\text { Mountain }\end{array}$ & -- do- & $\begin{array}{l}\text { T. } 28 \mathrm{~S} ., \\
\mathrm{R} .4 \mathrm{E} .\end{array}$ & $\begin{array}{l}\text { Navajo } \\
\text { Sand stone }\end{array}$ & Jurassic & ss & -- & -- \\
\hline $9 \mathrm{~A}$ & $\begin{array}{l}{ }^{4} \text { Sweetwater } \\
\text { dome }\end{array}$ & $\begin{array}{l}\text { Emery, } \\
\text { Wayne }\end{array}$ & $\begin{array}{l}\mathrm{T} .26 \mathrm{~S} ., \\
\mathrm{R} .14 \mathrm{E} .\end{array}$ & $\begin{array}{l}\text { Entrada, } \\
\text { Curtis }\end{array}$ & $---d o-\cdots$ & ss & $\rightarrow$ & - \\
\hline $9 B$ & ${ }^{2}$ Nequola Arch & $---\mathrm{do}---$ & $\begin{array}{l}\text { T. } 25-28 \mathrm{~S} ., \\
\text { R. } 13-16 \mathrm{E} \text {. }\end{array}$ & $\begin{array}{l}\text { White Rim, } \\
\text { Moenkopi }\end{array}$ & $\begin{array}{l}\text { Permian, } \\
\text { Triassic }\end{array}$ & ss & --- & $35 *$ \\
\hline $10^{2}$, & $\begin{array}{l}\text {,5an Rafael } \\
\text { Swel1 } \\
\text { (10 occur.) }\end{array}$ & Emery & $\begin{array}{l}\text { T. } 20-26 \mathrm{~S} ., \\
\text { R. } 9-14 \mathrm{E} .\end{array}$ & $\begin{array}{l}\text { Moenkop1, } \\
\text { Moss Back } \\
\text { Member of Ch1 }\end{array}$ & $\begin{array}{l}\text { Triassic } \\
\text { nle }\end{array}$ & ss & $0-500$ & $14^{*}$ \\
\hline 11 & $\begin{array}{l}{ }^{1} \text { Ten Mile Wash } \\
\text { (near Salt } \\
\text { Wash) }\end{array}$ & Grand & $\begin{array}{l}\text { T. } 23,24 \mathrm{~S} ., \\
\text { R. } 18,19 \mathrm{E} .\end{array}$ & Entrada & Jurassic & ss & ${ }^{5} 0-500+$ & $5_{5-30}$ \\
\hline 12 & $\mathbf{l}_{\text {Sa1t Wash }}$ & $---d o---$ & $\begin{array}{l}{ }^{5} \mathrm{~T} .22,23 \mathrm{~S} . \\
\mathrm{R} .16,17 \mathrm{E} .\end{array}$ & $\begin{array}{l}\text { Salt Wash } \\
\text { Member of } \\
\text { Morrison }\end{array}$ & Jurassic & --- & $50-500+$ & $5_{5-30}$ \\
\hline 13 & ${ }^{2} \mathrm{P} \cdot \mathrm{R} \cdot$ Spring & $\begin{array}{l}\text { Grand, } \\
\text { Uintah }\end{array}$ & T. 12-17 S., & Green River & Eocene & $\begin{array}{l}5 \text { ss, } \\
\text { sts }\end{array}$ & $0-300$ & $39 *$ \\
\hline 14 & $2_{\text {Hi } 11 \text { Creek }}$ & Uintah & $\begin{array}{l}\text { T. } 13-15 \mathrm{~S} ., \\
\text { R. } 19-21 \mathrm{E} .\end{array}$ & $---d o---$ & --- do- & $\begin{array}{l}5 \text { ss, } \\
\text { sts }\end{array}$ & $0-400$ & $25 *$ \\
\hline $15 \mathrm{~A}$ & ${ }^{2}$ Sunnyside & Carbon & $\begin{array}{l}\text { T. } 12-14 \mathrm{~S} ., \\
\text { R. } 13-15 \mathrm{E} .\end{array}$ & $\begin{array}{l}\text { Green River, } \\
\text { Colton }\end{array}$ & ---do--- & $\begin{array}{l}\text { Sss }_{\text {s }} \\
\text { sts }\end{array}$ & $0-500$ & $230 \star$ \\
\hline $15 B$ & $\begin{array}{l}{ }^{2} \text { Cottonwood- } \\
\text { Jacks Canyon }\end{array}$ & $\begin{array}{l}\text { Carbon, } \\
\text { Duchesne }\end{array}$ & $\begin{array}{l}{ }^{5} \mathrm{~T} \cdot 11-13 \mathrm{~S} ., \\
\mathrm{R} \cdot 14-17 \mathrm{E} .\end{array}$ & Green River & $---\mathrm{do}---$ & $\begin{array}{l}{ }^{5} \text { ss, } \\
\text { sts }\end{array}$ & -- & --- \\
\hline
\end{tabular}




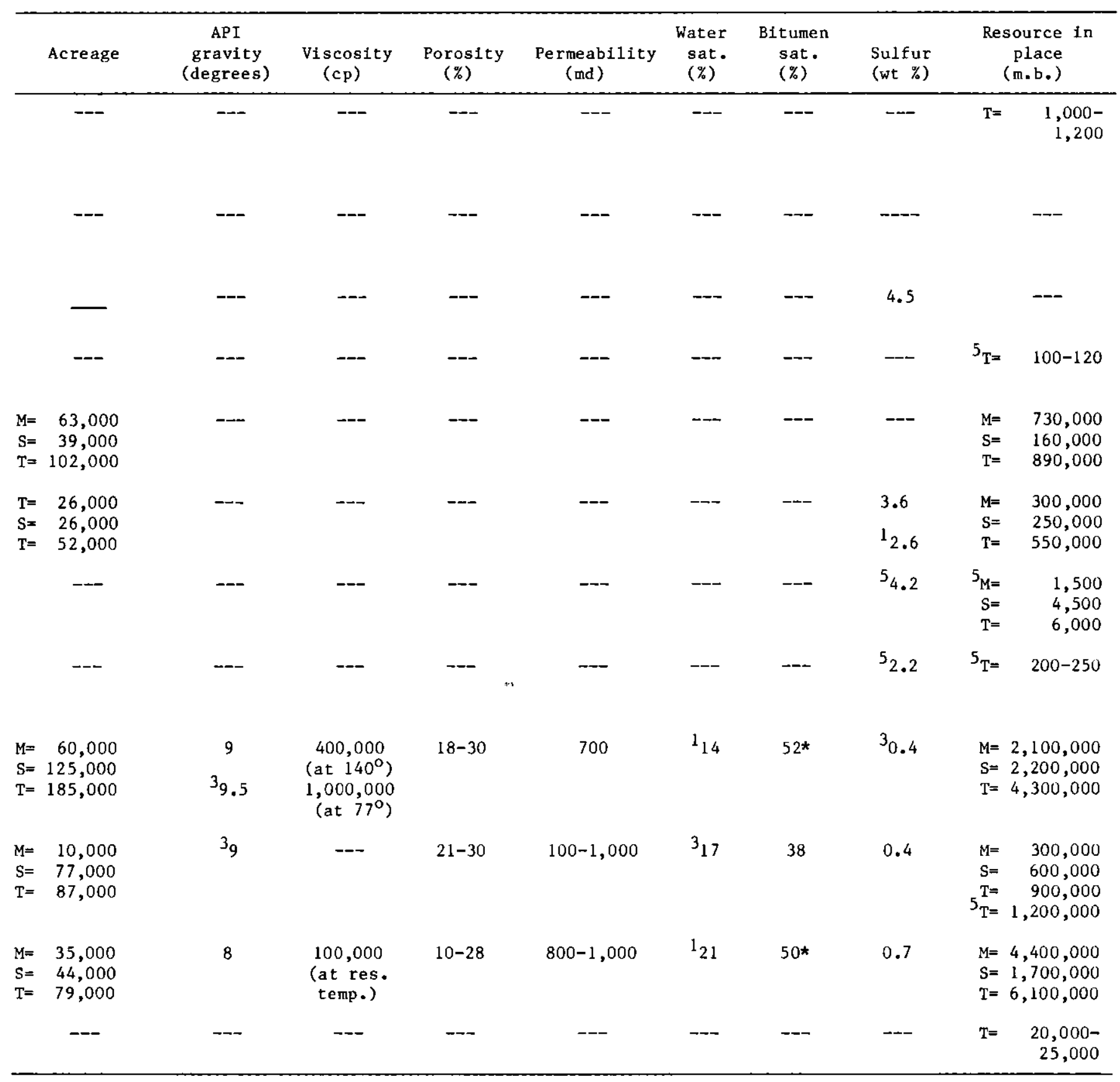


Table 16. Summary of bitumen deposits in Utah-Continued

\begin{tabular}{|c|c|c|c|c|c|c|c|c|}
\hline $\begin{array}{l}\text { Map } \\
\text { number }\end{array}$ & Deposit name & County & $\begin{array}{l}\text { Townships } \\
\text { and Ranges }\end{array}$ & Formation & Age & $\begin{array}{l}\text { Lith- } \\
\text { ology }\end{array}$ & $\begin{array}{l}\text { Depth } \\
(\mathrm{ft})\end{array}$ & $\begin{array}{l}\text { Pay } \\
\text { thickness } \\
\quad(\mathrm{ft})\end{array}$ \\
\hline $17 \mathrm{~A}$ & ${ }^{2}$ W11low Creek & $\begin{array}{l}\text { Duchesne, } \\
\text { Utah, } \\
\text { Wasatch }\end{array}$ & $\begin{array}{l}\text { T. } 11 \text { S., } \\
\text { R. } 9,10 \mathrm{E} . ; \\
\text { T. } 6,7, \mathrm{~S} ., \\
\text { R. } 8,9, \mathrm{~W} .\end{array}$ & $---\mathrm{do}=--$ & $\rightarrow-$ do- -- & ${ }^{5} \mathrm{ss}$, & -- & --- \\
\hline $17 \mathrm{~B}$ & $\begin{array}{l}{ }^{2} \text { Minnie Maude } \\
\text { Creek }\end{array}$ & $\begin{array}{l}\text { Carbon, } \\
\text { Duchesne }\end{array}$ & $\begin{array}{l}{ }^{5} \cdot 11,12 \mathrm{~s} ., \\
\mathrm{T} .11-13 \mathrm{E} .\end{array}$ & $---\mathrm{do}---$ & $---\mathrm{do} 0--$ & $\begin{array}{l}5_{s s,} \\
\text { sts, 1s }\end{array}$ & $0-500+$ & $5_{5-15}$ \\
\hline $17 \mathrm{C}$ & ${ }^{2}$ Argyle Canyon & Duchesne & $\begin{array}{l}{ }^{5} \mathrm{~T} .10,11 \mathrm{~S} ., \\
\mathrm{R} .11-13 \mathrm{E} . ; \\
\mathrm{T} .7 \mathrm{~S} . \\
\text { R. } 7 \mathrm{~W} .\end{array}$ & $---\mathrm{do}--$ & $---\mathrm{do}=--$ & ss & $5_{0-500+}$ & $515-60$ \\
\hline 19 & $\begin{array}{l}{ }^{5} \text { Daniels } \\
\text { Canyon }\end{array}$ & Wasatch & $\begin{array}{l}\text { T. } 6 \mathrm{~S} ., \\
\text { R. } 6 \mathrm{E} .\end{array}$ & Oquirrh & $\begin{array}{l}\text { Permian- } \\
\text { Pennsy1- } \\
\text { vanian }\end{array}$ & $\begin{array}{l}\text { Is } \\
\text { qtz }\end{array}$ & --- & --- \\
\hline 20 & ${ }^{5}$ Tabiona & Duchesne & $\begin{array}{l}\text { T. } 1 \text { S., } \\
\text { R. } 7 \text { W. }\end{array}$ & $\begin{array}{l}\text { Currant } \\
\text { Creek } \\
\text { Duchesne } \\
\text { River }\end{array}$ & $\begin{array}{l}\text { Paleocene- } \\
\text { Eocene, } \\
\text { Eocene }\end{array}$ & ss & $0-400$ & $5-150$ \\
\hline 21 & ${ }^{5}$ Lake Fork & $---\mathrm{do}---$ & $\begin{array}{l}\text { T. I N. } \\
\text { R. 4, 5' W. }\end{array}$ & $\begin{array}{l}\text { Duchespe } \\
\text { River }\end{array}$ & Eocene & ss & $0-450$ & $5-70$ \\
\hline 23 & ${ }^{2}$ White Rocks & $-\infty-d_{0}--$ & $\begin{array}{ll}\mathrm{T} . & 2 \mathrm{~N} ., \\
\mathrm{R} .1 & \mathrm{~W} ., 1 \mathrm{E} .\end{array}$ & Navajo & Jurassic & ss & $14-130$ & $500 *$ \\
\hline 24 & $\begin{array}{l}{ }^{2} \text { Asphalt/ } \\
\text { Ridge } \\
\text { Asphalt Ridge } \\
\text { N.W. }\end{array}$ & $---d 0^{---}$ & $\begin{array}{l}\text { T. } 4-6 \text { S. } \\
\text { R. } 20-22 \text { E. }\end{array}$ & $\begin{array}{l}\text { Mesaverde } \\
\text { Group (Rim- } \\
\text { rock Sandstone) }\end{array}$ & Cretaceous & ss & $20-600$ & $40 *$ \\
\hline
\end{tabular}




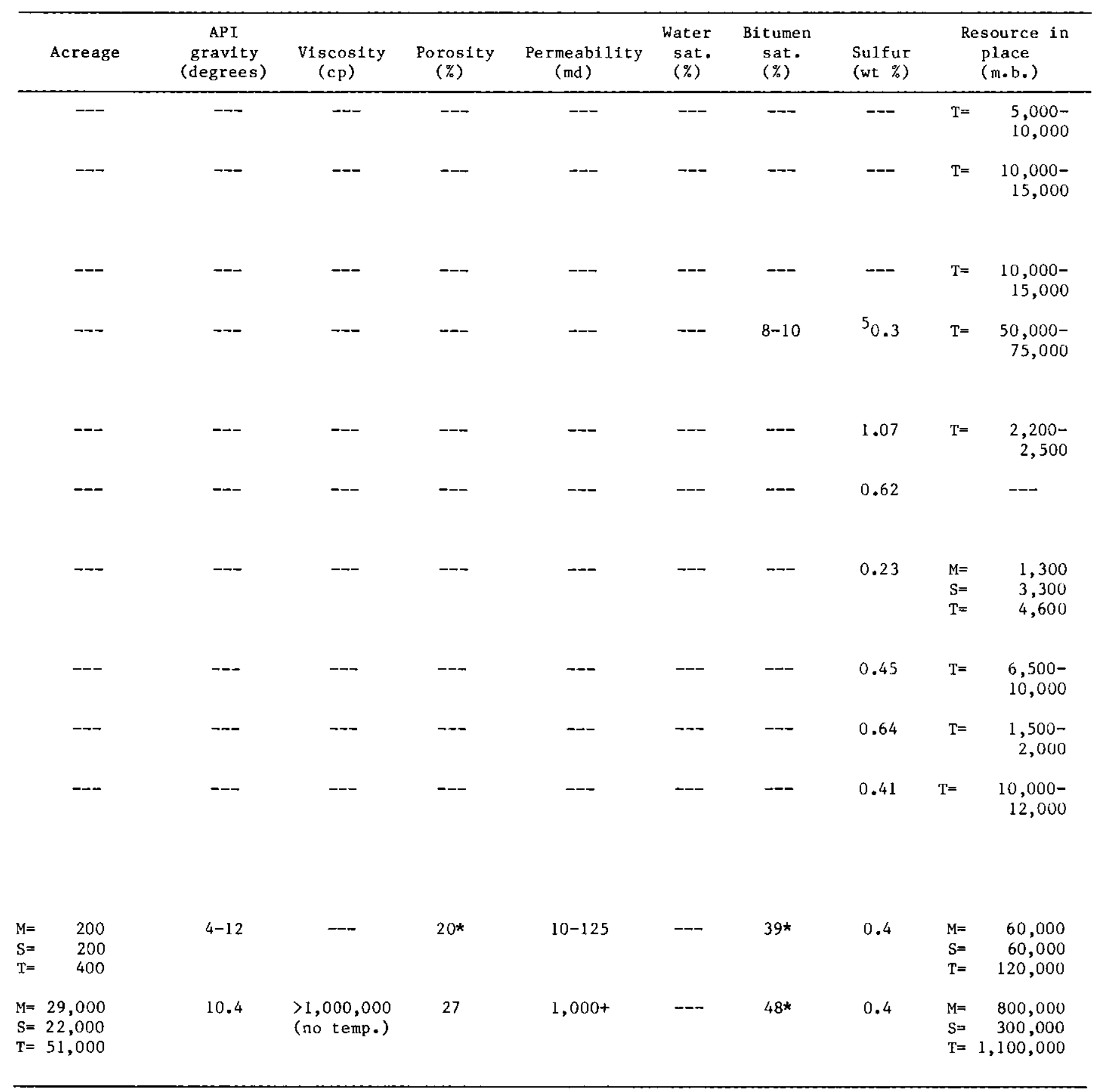


Table 16. Summary of bitumen deposits in Utah-Continued

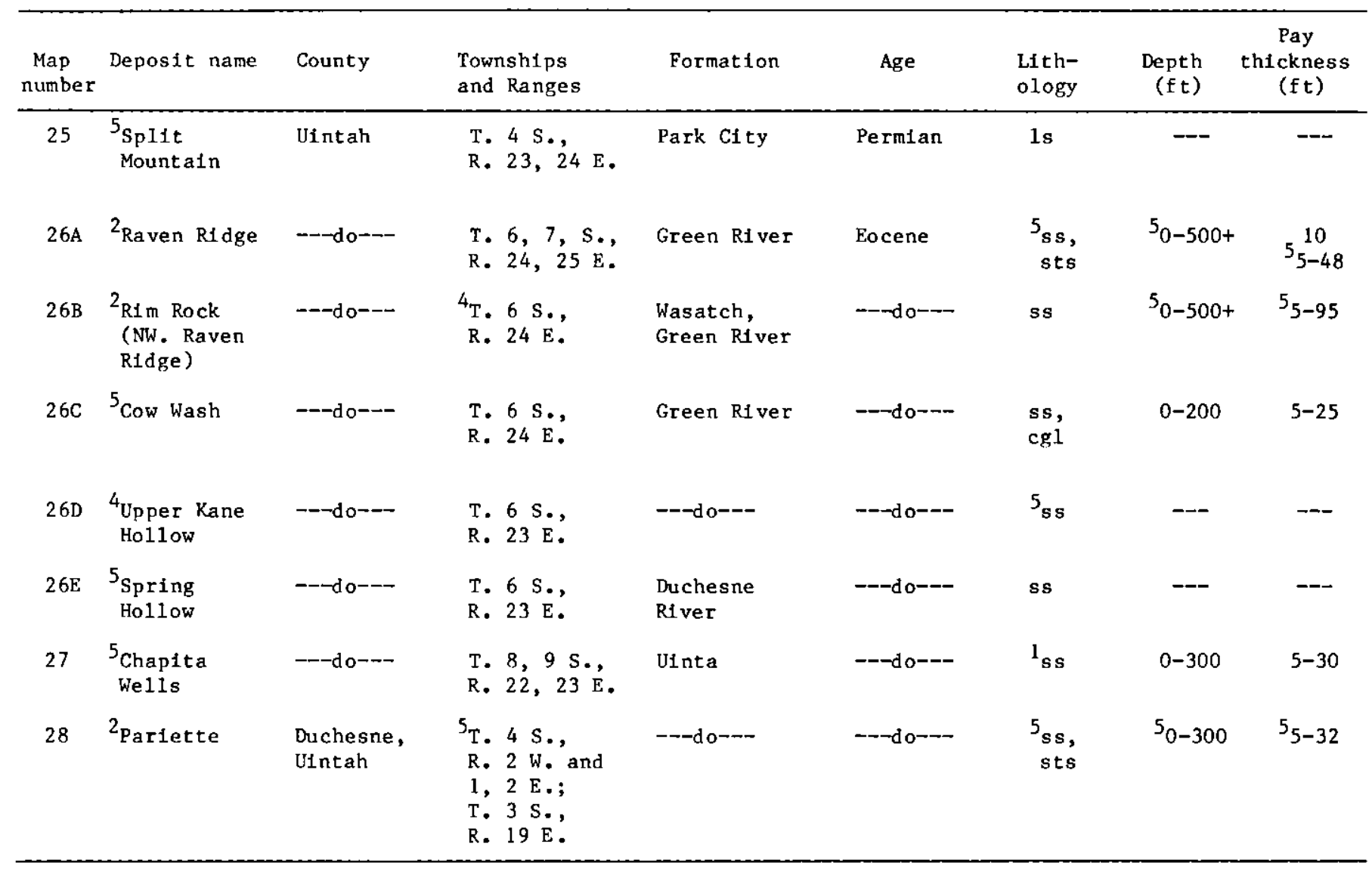




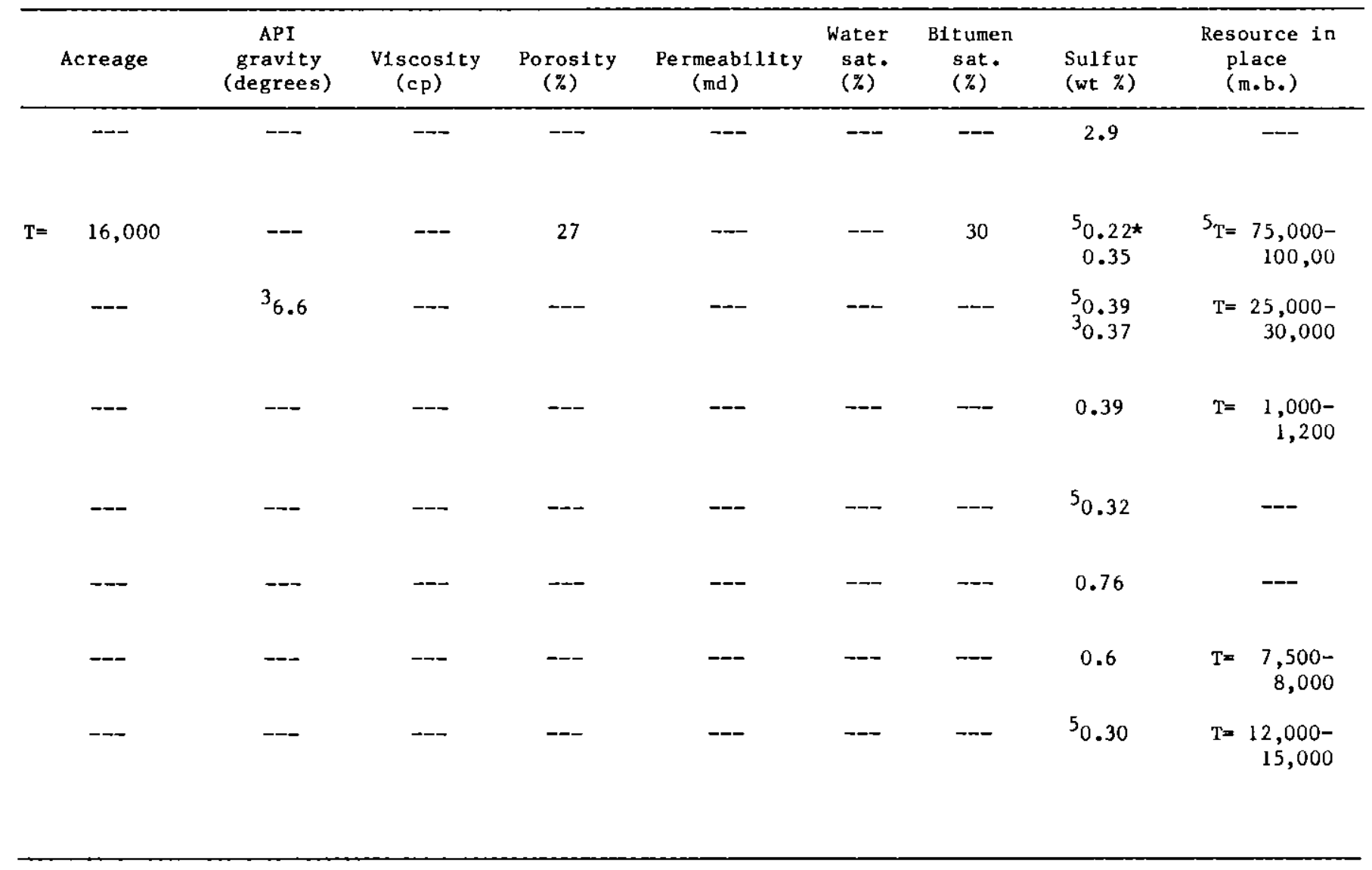


Table 17. Summary of bitumen deposits in Wyoming

\begin{tabular}{|c|c|c|c|c|c|c|c|c|}
\hline $\begin{array}{l}\text { Map } \\
\text { number }\end{array}$ & Deposit name & County & $\begin{array}{l}\text { Townships } \\
\text { and Ranges }\end{array}$ & Formation & Age & $\begin{array}{l}\text { Lith- } \\
\text { ology }\end{array}$ & $\begin{array}{l}\text { Depth } \\
(\mathrm{ft})\end{array}$ & $\begin{array}{l}\text { Pay } \\
\text { thickness } \\
\quad(f t)\end{array}$ \\
\hline 1 & $\begin{array}{l}{ }^{2} \text { Burnt Hollow } \\
\text { ( } 2 \text { areas) }\end{array}$ & Crook & $\begin{array}{l}\text { T. } 54,55 \mathrm{~N} ., \\
\text { R. } 63,64 \mathrm{~W} .\end{array}$ & Minnelusa & Permian & ss & $\begin{array}{l}600- \\
1,000\end{array}$ & $35-45 \star$ \\
\hline 2 & $\begin{array}{l}1_{\text {Rocky Ford, }} \\
\text { Beulah }\end{array}$ & ---do--- & $\begin{array}{l}\text { T. } 52 \mathrm{~N} ., \\
\text { R. } 60,61 \mathrm{w} .\end{array}$ & $---\mathrm{do}-\longrightarrow$ & Pennsylvanian & ss & 100 & --- \\
\hline 3 & $\begin{array}{l}{ }^{1} \text { Oil Butte, } \\
\text { Moorcroft }\end{array}$ & --- do--- & $\begin{array}{l}\text { T. } 51,52 \mathrm{~N} ., \\
\text { R. } 67 \mathrm{~W} .\end{array}$ & $\begin{array}{l}\text { Muddy } \\
\text { Sandstone, } \\
\text { Mowry Shale, } \\
\text { Dakota } \\
\text { Sandstone }\end{array}$ & Cretaceous & $\begin{array}{l}\text { ss, } \\
\text { sh }\end{array}$ & outcrop & -- \\
\hline 4 & ${ }^{1}$ Thornton & Weston & $\begin{array}{l}\text { T. } 48 \mathrm{~N} ., \\
\text { R. } 66 \mathrm{~W} .\end{array}$ & $\begin{array}{l}\text { Turner Sandy } \\
\text { Member of } \\
\text { Car1isle Shale }\end{array}$ & $---d o---$ & $\mathrm{ss}$ & --- & --- \\
\hline 5 & ${ }^{\mathrm{l}}$ Newcastle & $---\mathrm{do}=-$ & $\begin{array}{l}\text { T. } 44,45 \mathrm{~N}_{\bullet}, \\
\mathrm{R} .6 \mathrm{I}, 62 \mathrm{~W} \cdot\end{array}$ & Newcastle & $---\mathrm{do}---$ & ss & -- & --- \\
\hline 6 & ${ }^{1}$ Salt Creek & Natrona & $\begin{array}{l}\text { T. } 40 \text { N., } \\
\text { R. } 79 \text { W. }\end{array}$ & Shannon & ---do-- & ss & --- & 8 \\
\hline 7 & $\begin{array}{l}\mathrm{l}_{\text {Tisdale }} \\
\text { anticline }\end{array}$ & $\begin{array}{l}\text { Johnson, } \\
\text { Natrona }\end{array}$ & $\begin{array}{l}\text { T. } 41 \mathrm{~N} ., \\
\text { R. } 81 \text { W. }\end{array}$ & $\begin{array}{l}\text { Morrison, } \\
\text { Sundance, } \\
\text { Cloverly }\end{array}$ & $\begin{array}{l}\text { Jurassic } \\
\text { Cretaceous }\end{array}$ & ss & - & $6-7$ \\
\hline 8 & $\begin{array}{l}{ }^{1} \text { Sheep Mtn. } \\
\text { Canyon }\end{array}$ & Big Horn & $\begin{array}{l}\text { T. } 54 \mathrm{~N} ., \\
\text { R. } 94 \mathrm{~W} .\end{array}$ & Madison & Mississippian & 1s & --- & -- \\
\hline $9 \mathrm{~A}$ & $1_{\text {Red Gulch }}$ & $---\mathrm{do}^{---}$ & $\begin{array}{l}\text { T. } 52 \mathrm{~N} ., \\
\text { R. } 90 \mathrm{~W} \text {. }\end{array}$ & $\begin{array}{l}\text { Tensleep, } \\
\text { Phosphoria }\end{array}$ & $\begin{array}{l}\text { Pennsylvanian- } \\
\text { Permian }\end{array}$ & ss & $\begin{array}{l}10 \text { to } \\
\text { outcrop }\end{array}$ & 10 \\
\hline $9 \mathrm{~B}$ & $\begin{array}{l}\text { Battle } \\
\text { Creek }\end{array}$ & $---\mathrm{do}---$ & $\begin{array}{l}\text { T. } 52 \mathrm{~N} ., \\
\text { R. } 89 \mathrm{~W} .\end{array}$ & Tensleep & $\begin{array}{l}\text { Pennsyl- } \\
\text { vanian }\end{array}$ & ss & -- & 6 \\
\hline 10 & ${ }^{\mathrm{I}_{\text {Ekay }} \text { Creek }}$ & Natrona & $\begin{array}{l}\text { T. } 38 \mathrm{N.}, \\
\text { R. } 87 \text { W. }\end{array}$ & Frontier & Cretaceous & ss & --- & --- \\
\hline $11 \mathrm{~A}$ & ${ }^{1}$ Cedar Ridge & Fremont & $\begin{array}{l}\text { T. } 39 \mathrm{~N} ., \\
\text { R. } 90,9 \mathrm{i} \text { W. }\end{array}$ & $\begin{array}{l}\text { Overturned } \\
\text { beds along } \\
\text { a fault }\end{array}$ & $\begin{array}{l}\text { Cretaceous and } \\
\text { Eocene }\end{array}$ & tuff & --- & --- \\
\hline
\end{tabular}

${ }_{1}^{1}$ Ball Assoctates, 1965.

${ }^{2}$ Lewtn and Associates, 1984. 


\begin{tabular}{|c|c|c|c|c|c|c|c|c|}
\hline Acreage & $\begin{array}{c}\text { API } \\
\text { gravity } \\
\text { (degrees) }\end{array}$ & $\begin{array}{l}\text { Viscosity } \\
(c p)\end{array}$ & $\begin{array}{l}\text { Porosity } \\
(\%)\end{array}$ & $\begin{array}{l}\text { Permeability } \\
\text { (md) }\end{array}$ & $\begin{array}{c}\text { Water } \\
\text { sat. } \\
(\%)\end{array}$ & $\begin{array}{c}\text { Bitumen } \\
\text { sat. } \\
(\%)\end{array}$ & $\begin{array}{l}\text { Sulfur } \\
\text { (wt \%) }\end{array}$ & $\begin{array}{c}\text { Resource in } \\
\text { place } \\
\left(\mathrm{m} \cdot \mathrm{b}_{.}\right)\end{array}$ \\
\hline $\begin{array}{l}M=3,500 \\
S=1,000 \\
T=4,500\end{array}$ & $5-9$ & $\begin{array}{c}1,000,000 \\
\text { e } 53^{\circ} \mathrm{F} \\
10 @ 345^{\circ} \mathrm{F}\end{array}$ & 25 & $1,000-2,000$ & -- & $\begin{array}{ll}51 & \mathrm{~N} . \text { area } \\
34 \mathrm{~S} \text {. area }\end{array}$ & 06 & $\begin{array}{l}\mathrm{M}=120,000 \\
\mathrm{~S}=25,000 \\
\mathrm{~T}=145,000\end{array}$ \\
\hline-- & --- & --- & --- & --- & 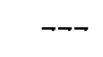 & --- & -- & -- \\
\hline--- & -- & $-\infty$ & -- & --- & --- & --- & -- & $\longrightarrow$ \\
\hline--- & --- & - & --- & $\cdots$ & --- & - & --- & --- \\
\hline--- & --- & --- & -- & - & --- & -- & --- & $\longrightarrow$ \\
\hline--- & --- & --- & --- & -- & --- & --- & -- & --- \\
\hline - - & --- & --- & -- & .-- & --- & --- & $\longrightarrow$ & -- \\
\hline--- & --- & -- & --- & $-\cdots$ & --- & --- & --- & --- \\
\hline--- & --- & --- & --- & -- & -- & --- & --- & --- \\
\hline--- & $\cdots$ & - & -- & -- & -- & -- & -- & -- \\
\hline -די & -- & -- & -- & -- & --- & -- & --- & - \\
\hline--- & --- & --- & --- & --- & --- & --- & --- & -- \\
\hline
\end{tabular}


Table 17. Summary of bitumen deposits in Wyoming - Continued

\begin{tabular}{|c|c|c|c|c|c|c|c|c|}
\hline $\begin{array}{l}\text { Map } \\
\text { number }\end{array}$ & Deposit name & County & $\begin{array}{l}\text { Townships } \\
\text { and Ranges }\end{array}$ & Formation & Age & $\begin{array}{l}\text { Lith- } \\
\text { ology }\end{array}$ & $\begin{array}{l}\text { Depth } \\
(\mathrm{ft})\end{array}$ & $\begin{array}{c}\text { Pay } \\
\text { thickness } \\
(f t)\end{array}$ \\
\hline $11 B$ & ${ }^{1}$ Dry Creek & Fremont & $\begin{array}{l}\text { T. } 39 \mathrm{~N}_{.}, \\
\mathrm{R} .92,93, \mathrm{~W} .\end{array}$ & $\begin{array}{l}\text { overturned } \\
\text { beds along } \\
\text { a fault }\end{array}$ & Eocene & $\begin{array}{l}\text { tuff, } \\
\text { ss }\end{array}$ & --- & -- \\
\hline $12 \mathrm{~A}$ & $\begin{array}{l}{ }_{\text {Alkalat }} \\
\text { Butte }\end{array}$ & $---\mathrm{do}---$ & $\begin{array}{l}\text { T. } 34 \text { N., } \\
\text { R. } 95 \mathrm{~W} . ; \\
\text { T. } 1,2 \text { S. } \\
\text { R. } 6 \text { E. }\end{array}$ & $\begin{array}{l}\text { Cody Shale, } \\
\text { Mesaverde, } \\
\text { Fort Unton, } \\
\text { Wind River }\end{array}$ & $\begin{array}{l}\text { Cretaceous, } \\
\text { Paleocene, } \\
\text { Eocene }\end{array}$ & $\begin{array}{l}\text { ss, } \\
\text { cg1 }\end{array}$ & outcrop & -- \\
\hline $12 \mathrm{~A}$ & $\mathrm{l}_{\text {Muskrat }}$ & --- do--- & $\begin{array}{l}\text { T. } 34 \mathrm{~N} ., \\
\text { R. } 92 \text { W., } \\
\text { secs. } 17,18\end{array}$ & Fort Union & Paleocene & $\begin{array}{l}\text { ss, } \\
\text { cg1 }\end{array}$ & -- & -- \\
\hline $13^{2}$, & $\begin{array}{l}{ }^{3} \text { Rattlesnake } \\
\text { H1lls }\end{array}$ & Natrona & $\begin{array}{l}{ }_{\mathrm{T}} \cdot 33 \mathrm{~N} . \\
\mathrm{R} \cdot 86-88 \mathrm{~W} .\end{array}$ & Muddy & Cretaceous & $\begin{array}{l}\text { ss, } \\
\text { cg1 }\end{array}$ & $0-1,400$ & $38 *$ \\
\hline 14 & $\begin{array}{l}{ }^{1} \text { Dutton } \\
\text { Basin }\end{array}$ & Fremont & $\begin{array}{l}\text { T. } 33 \mathrm{~N} ., \\
\text { R. } 90 \text { W., } \\
\operatorname{secs} 33,12 \text {, } \\
22,24\end{array}$ & $\begin{array}{l}\text { Chugwater, } \\
\text { Frontier, } \\
\text { Wind River }\end{array}$ & $\begin{array}{l}\text { Triassic, } \\
\text { Cretaceous, } \\
\text { Eocene }\end{array}$ & ss & -- & --- \\
\hline 15 & $\begin{array}{l}{ }^{1} \text { Conant } \\
\text { Creek }\end{array}$ & $---\mathrm{do}^{-}--$ & $\begin{array}{l}\text { T. } 32 \mathrm{~N} ., \\
\text { R. } 94 \mathrm{~W} . \text {, } \\
\text { secs. } 2-5 \text {, } \\
10,11\end{array}$ & unnamed & Eocene & $\begin{array}{l}\text { ss, } \\
\text { cgI }\end{array}$ & -- & - \\
\hline I $6 \mathrm{~A}$ & ${ }^{1}$ Dallas & $---\mathrm{do}---$ & $\begin{array}{l}\text { T. } 32 \mathrm{~N} ., \\
\text { R. } 99 \text { W., } \\
\text { secs. } 13,24\end{array}$ & Chugwater & Triassic & -- & -- & -- \\
\hline $16 \mathrm{~B}$ & $\begin{array}{l}1_{\text {Lettle Popo }} \\
\text { Agle }\end{array}$ & $\rightarrow \mathrm{do}^{--}$ & $\begin{array}{l}\text { T. } 31 \text { N., } \\
\text { R. } 99 \text { W., } \\
\text { sec. } 8\end{array}$ & Phosphorta & Permian & ss & -- & $5-10$ \\
\hline 17 & $\begin{array}{l}\text { l Lime }_{\text {Creek }} \\
\text { Crees }\end{array}$ & Sublette & $\begin{array}{l}\mathrm{T} .38 \mathrm{~N} . \\
\mathrm{R} .110 \mathrm{~W} .\end{array}$ & --- do- $^{-}$ & $\rightarrow-\mathrm{do}-\cdots$ & ss & -- & -- \\
\hline 18 & $\begin{array}{l}1_{\text {Muddy }} \\
\text { Creek }\end{array}$ & Carbon & $\begin{array}{l}\text { T. } 17 \text { N., } \\
\text { R. } 92 \text { w., } \\
\text { secs. 3, } 10, \\
15,16,22,27\end{array}$ & $\begin{array}{l}\text { Fort Union, } \\
\text { Wasatch }\end{array}$ & $\begin{array}{l}\text { Paleocene, } \\
\text { Eocene }\end{array}$ & $\begin{array}{l}\text { ss, } \\
\text { cg1 }\end{array}$ & -- & $0-50$ \\
\hline 19 & ${ }^{1}$ Shell Point & Sweetwater & $\begin{array}{l}\text { T. } 12 \text { N., } \\
\text { R. } 97 \text { W., } \\
\text { sec. } 9\end{array}$ & Green River & Eocene & ss & $--\infty$ & (thin) \\
\hline 20 & ${ }^{1}$ Sterra Madre & Carbon & $\begin{array}{l}\text { T. } 12-14 \mathrm{~N}_{.}, \\
\text {R. } 86,87 \text { W. }\end{array}$ & Madison & Missippian & $1 \mathrm{~s}$ & outcrop & patches \\
\hline
\end{tabular}




\begin{tabular}{|c|c|c|c|c|c|c|c|c|}
\hline Acreage & $\begin{array}{c}\text { API } \\
\text { gravity } \\
\text { (degrees) }\end{array}$ & $\begin{array}{l}\text { Viscosity } \\
(c n)\end{array}$ & $\begin{array}{l}\text { Porosity } \\
(\%)\end{array}$ & $\begin{array}{l}\text { Permeability } \\
\quad \text { (md) }\end{array}$ & $\begin{array}{l}\text { Water } \\
\text { sat. } \\
(\%)\end{array}$ & $\begin{array}{c}\text { B1tumen } \\
\text { sat. } \\
(\%)\end{array}$ & $\begin{array}{l}\text { Sulfur } \\
\text { (wt \%) }\end{array}$ & $\begin{array}{c}\text { Resource In } \\
\text { place } \\
(\mathrm{m} \cdot \mathrm{b} .)\end{array}$ \\
\hline--- & -- & $\therefore-$ & -- & $\cdots$ & -- & -- & -- & -- \\
\hline--- & $m$ & -- & $\sim$ & -- & -- & -- & --- & --- \\
\hline-- & -- & --- & -- & -- & -- & --- & --- & -- \\
\hline $\begin{array}{l}S=1,500 \\
T=1,500\end{array}$ & -- & $\begin{array}{c}12,000 \text { at } \\
\text { ? temp. }\end{array}$ & 25 & 1,000 & - & $41^{*}$ & --- & $\begin{array}{ll}S= & 45,000 \\
T= & 45,000\end{array}$ \\
\hline--- & - & --- & $-\cdots$ & --- & --- & --- & --- & -- \\
\hline--- & $-\infty$ & $\cdots$ & --- & --- & --- & - & -- & -- \\
\hline--- & --- & -- & -- & - & $\sim$ & $-\infty$ & $-\infty$ & --- \\
\hline$-\infty$ & -- & -- & -- & -- & --- & -- & --- & --- \\
\hline--- & -- & --- & --- & --- & - & $3.2 \%$ of1 & --- & - \\
\hline--- & -- & -- & --- & - & --- & --- & $\cdots$ & -- \\
\hline-- & -- & -- & $\cdots$ & -- & -- & --- & -- & --- \\
\hline--- & -- & -- & --- & -- & - & -- & -- & $-\infty$ \\
\hline
\end{tabular}







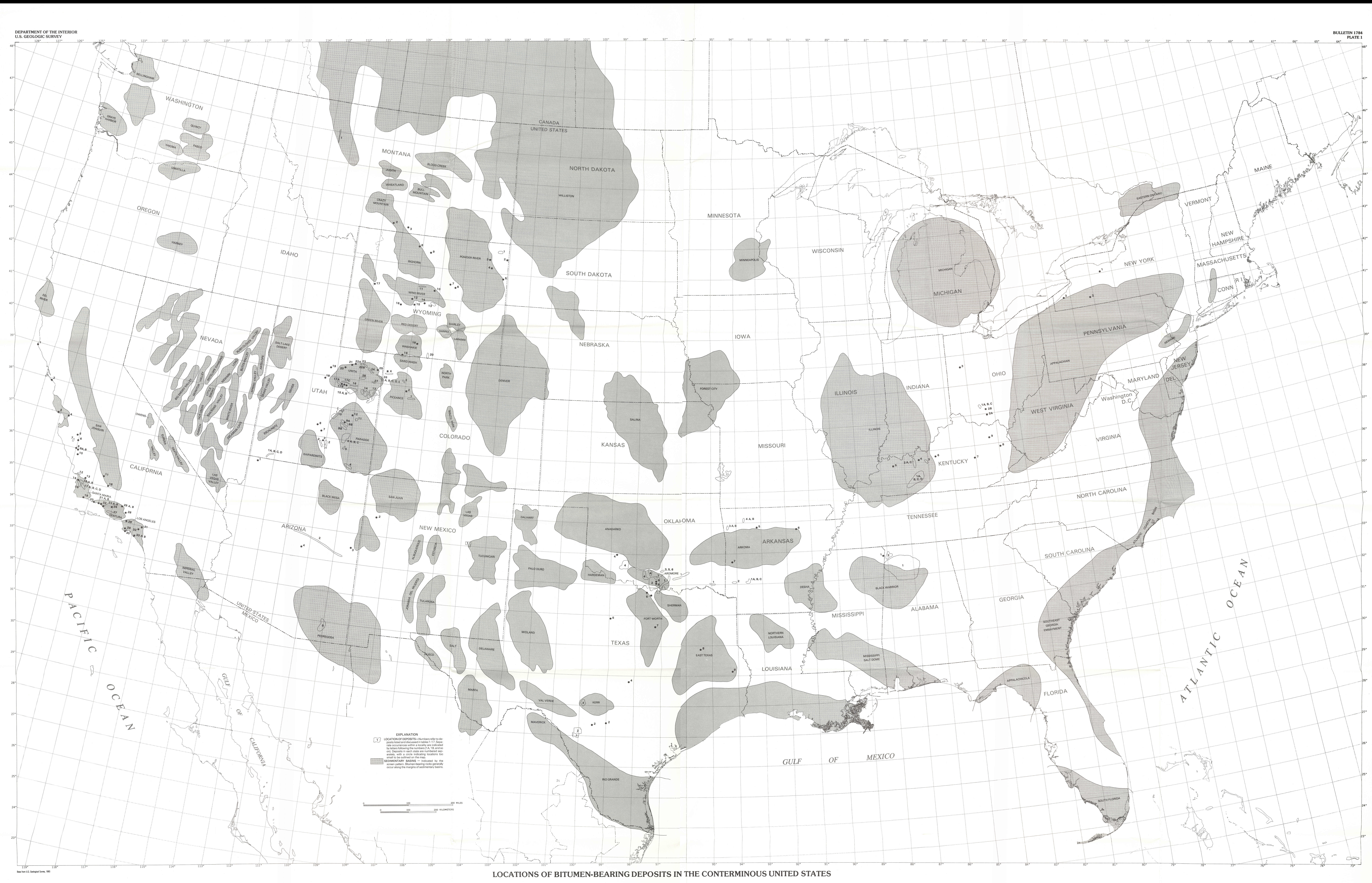




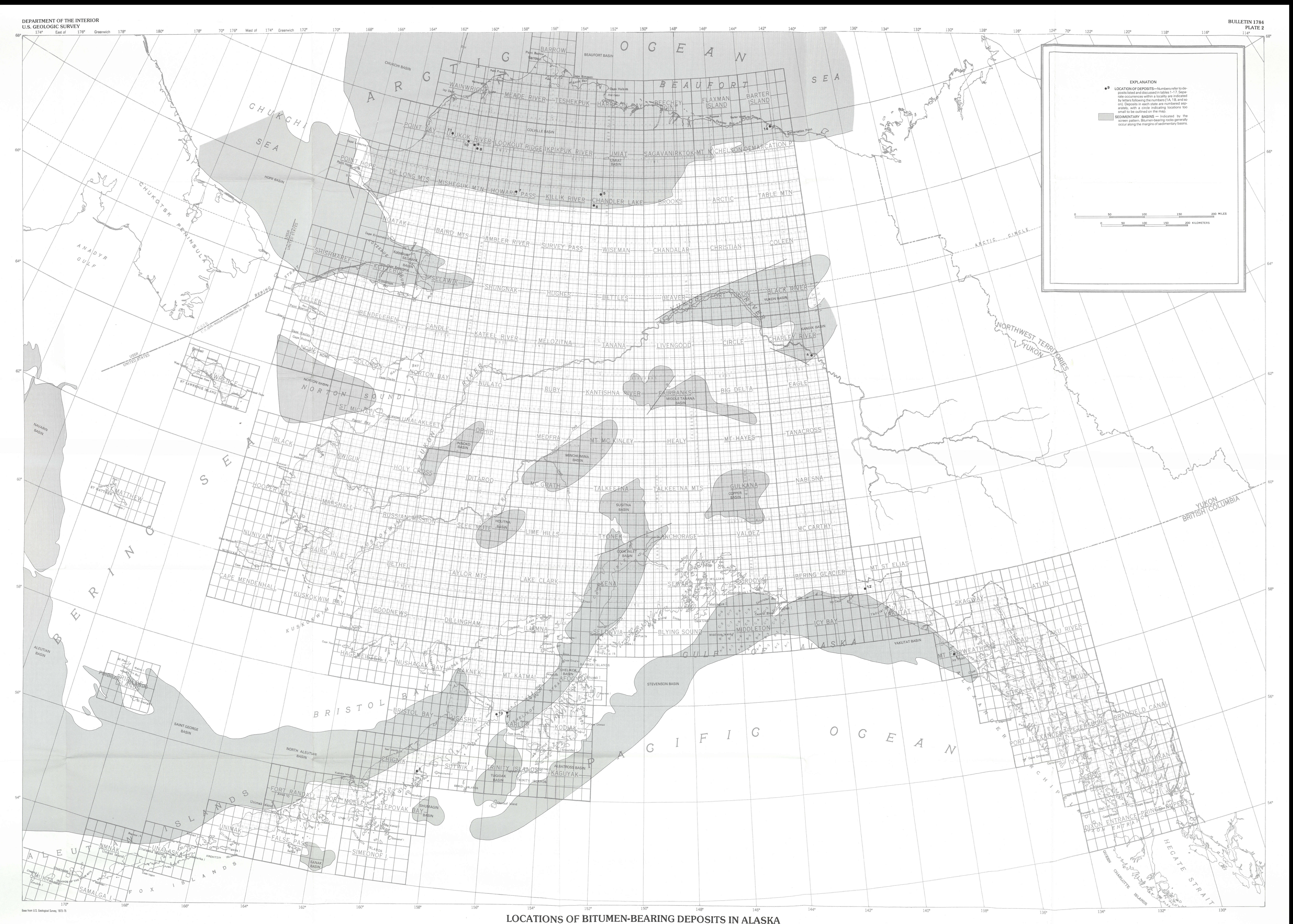

LOCATIONS OF BITUMEN-BEARING DEPOSITS IN ALASKA 
